\title{
Análisis de la Escena en la Cocina por Medio de Sensores loT Diseñados Basados en el Microcontrolador Node MCU ESP8266 y Conectados al Servidor ThingSpeak
}

\author{
Yeny Y. Muñoz ${ }^{(1)}$, Omar D. Castrillón ${ }^{(1)}$, Luis F. Castillo ${ }^{(1,2)}$ y Carlos López $^{(3)}$ \\ (1) Universidad Nacional de Colombia - Sede Manizales, Facultad de Ingeniería y Arquitectura, Departamento de \\ Ingeniería Industrial, Grupo de Innovación y Desarrollo Tecnológico, Bloque Q Campus La Nubia, Manizales, 170001 - \\ Colombia. (e-mail: odcastrillong@unal.edu.co,yymunozc@unal.edu.co) \\ (2) Universidad de Caldas, Facultad de Ingenierías, Manizales-Colombia. (e-mail: Ifcastilloos@unal.edu.co) \\ (3) MABE, Manizales-Colombia. (e-mail: Carlos.Lopez@mabe.com.co).
}

Recibido Nov. 17, 2018; Aceptado Ene. 4, 2019; Versión final Mar. 1, 2019, Publicado Oct. 2019

\begin{abstract}
Resumen
Este articulo presenta el diseño de diferentes sensores para la cocina usando como base el microcontrolador Node MCU ESP8266, y su comunicación con el servidor ThingSpeak, en primera medida se realizó la prueba de cada sensor para analizar su funcionamiento, luego se llevó a cabo el diseño de las PCB por medio del programa PROTEUS, después se efectuó el montaje electrónico, y por último se implementó la comunicación con el servidor ThingSpeak vía wifi, la cual permitió ver diferentes gráficas de los estados de los sensores en intervalos de 10 segundos, y realizar el análisis de la escena en la cocina. Se concluye que el sistema tiene un porcentaje del $100 \%$ de eficiencia cuando se usa el sensor de magnético de puerta, el sensor de corriente y el sensor de flujo de agua, y tiene una efectividad del $86,2 \%$ respecto al sensor de movimiento, y el análisis realizado por medio gráficas adquiridas en el servidor ThingSpeak fue óptimo dado que se pudo establecer la cantidad de datos adquiridos, un intervalo de tiempo aproximado de duración de la adquisición de datos, y una fecha exacta donde los sensores cambiaron de estado.
\end{abstract}

Palabras clave: sensores; cocina; escena; ESP8166; Internet de las cosas

\section{Kitchen Scene Environment Analysis via loT sensors, Designed with a MCU ESP8266 Node Microcontroller and Connected to the ThingSpeak Server}

\begin{abstract}
This article presents the design of several sensors used in a kitchen environment, using a MCU ESP8266 node microcontroller as a base, which communicates with the ThingSpeak server. Firstly, each sensor was tested to analyze its functioning, then PCBs were designed using PROTEUS; afterwards, electronic circuit was designed and mounted and finally, communication with the server was implemented via Wi-Fi. This allowed to determine different states of the sensor by 10 seconds intervals and to analyze the kitchen scene setting. It is concluded that the system is $100,0 \%$ efficient when magnetic door, current and water flow sensors are used; and displays an $86,2 \%$ efficiency with the movement sensor and the analysis via acquired graphics in the ThingSpeak server showed optimal results because the amount and time interval of data acquisition and date of sensors state change, were established accordingly.
\end{abstract}

Keywords: sensors; kitchen; scene; ESP8266; Internet of Things 


\section{INTRODUCCIÓN}

La cocina es el lugar más importante de cada uno de los hogares, dado que allí se prepara el alimento que se degusta diariamente. En la actualidad, se ha venido automatizando el hogar, en torno a la iluminación inteligente, la calefacción, la ventilación, enfocándolo a la reducción del consumo de energía (Parsch y Masrur, 2017), se han realizado sistemas inteligentes para el uso eficiente de unidades sanitarias (Gonzales et al., 2017), la automatización busca transferir las tareas realizadas por los humanos, a un conjunto de elementos tecnológicos (Toloza, 2016), es así como se observa que se han creado prototipos-robots, los cuales buscan que operen en entornos humanos estándar, automatizando tareas comunes siguiendo el paradigma de robótica ubicua (Bogdan et al., 2008). Otra vertiente es la de proyectos donde la finalidad de la aplicación domótica es la de ayudar a personas con necesidades especiales (Rodríguez, 2010). Hoy en día existe un auge relacionado con el internet de las cosas (IoT), el cual es una plataforma donde cada día los dispositivos se vuelven más inteligentes y existen diferentes arquitecturas loT enfocadas a resolver problemas de la vida real (Ray, 2018); según SITRACK, una empresa de alta tecnología, dedicada a la provisión de servicios de información a la medida, ubicada en varios países como Argentina, Brasil, Chile, México, Uruguay, estima que para el 2020 habrá 24 mil millones de dispositivos loT, de los cuales 646 millones estarán relacionados con la salud, 310 millones serán usados por empresas de comidas y 75 millones con fines agrícolas (Sitrack, 2019), aunque se estima que los usuarios no son conscientes de como el loT afecta su privacidad y calidad de vida en general (Pia, 2019).

Según la literatura, el Internet de las cosas es una herramienta, la cual puede potencializar muchas áreas de la vida cotidiana como la salud, en la cual se busca conectar nanodispositivos a internet para realizar una detección y diagnóstico más temprana de enfermedades (Maksimović, 2017), la agricultura de precisión, para obtención, monitoreo y análisis de variables climáticas (Quiroga, et al., 2017), la seguridad, en la cual se busca implementar herramientas para analizar y proteger la seguridad de los sistemas loT distribuidos denominados SloT (Teixeira et al., 2019), entre otras (Castro et al., 2017); así mismo se están desarrollando modelos de negocios para productos y servicios basados en el loT (Klein et al., 2017) como, por ejemplo, servicios bibliotecarios para realizar el seguimiento de libros u otros textos (Bayani et al., 2018), se está tratando de implementar la teoría de la entropía, para describir la distribución y el poder de la electrónica en el loT (Wang, 2019), de igual manera se están desarrollando arquitecturas como la SmartCom, la cual busca monitorear y administrar viviendas mediante el uso de loT (Oliveira et al., 2017), adicionalmente se están explorando mejoras en el ámbito de transmisión de datos desde la estación base a nodos destino denominada multidifusión y así poder implementar nuevos protocolos como el de algortimos heurísticos (Li et al., 2013), igualmente se indaga cómo implementar tecnologías como BlockChain, la cual permite tener registros de datos en diferentes ubicaciones y es resistente a la manipulación indebida para poder dar seguridad en la transferencia de datos en las redes loT (Wang, 2019).

El incremento del loT conlleva a un incremento de volúmenes de datos, el desarrollo de métodos para analizar toda esta información es lo que se conoce como Big Data (Plasencia y Anias, 2016), métodos como el MapReduce buscan obtener un alto nivel de extracción, usando de manera ordenada funciones map, siendo reducidas en un entorno distribuido (Vidal et al., 2017). Así mismo se están creando plataformas como la SMASH con la capacidad de almacenar, consultar, analizar grandes volúmenes de datos, con el propósito de respaldar la toma de decisiones en la industria (Wilcox et al., 2019), y todo lo relacionado con loT y Big Data es un tema que demanda conocimiento y tecnología, y muchas empresas u organizaciones no pueden ofrecer soluciones basadas en loT (Sánchez y Ramoscelli, 2018), es así como lo demuestra un estudio realizado a 39 entidades públicas, ubicadas en la región cafetera de Colombia, donde se evidencia que no hay avance en el uso de tecnologías (Gómez et al, 2018), lo cual es una situación crítica dado que diversos estudios demuestran que la inversión en tecnologías de la información influye de manera positiva en la productividad (Arévalo et al., 2018), por lo tanto se busca aprovechar estos recursos del Big Data para mitigar el fracaso empresarial (Amankwah y Adomako, 2019).

En nuestro país aún no hay claridad sobre el número de dispositivos por sectores conectados a internet; sin embargo, se infiere del informe trimestral TIC que elabora el Mintic (Ministerio de Tecnologías de Información y Comunicaciones, 2014), que las suscripciones a internet continúan en aumento, especialmente en los estratos socioeconómicos bajos; desde esta perspectiva, se puede entonces deducir que la tendencia continuará y Colombia tendrá en el futuro cercano una de las tasas más altas de penetración de internet en los hogares, en lo que a Latinoamérica se refiere, es por eso que la investigación realizada en este artículo es de suma importancia, ya que se diseñaron sensores loT para los hogares, buscando a futuro el desarrollo de un producto mínimo viable y generando así oportunidades de negocio.

Por último, la amplia gama de opciones que brinda el Internet de las Cosas permitirá al sector público y privado obtener grandes resultados gracias al aprovechamiento de las investigaciones en este campo, mejorando la competitividad o diseño de nuevos productos y servicios que resuelvan problemas de la comunidad, la 
industria y las organizaciones, es por eso que el objetivo de esta investigación es diseñar sensores loT basados en el microcontrolador Node MCU ESP8266, el cual tiene un módulo wifi que permitirá conectar a través de internet distintos sensores como son: el sensor magnético de puerta MC-38, sensor de corriente ACS712, sensor de flujo de agua YF-S201, sensor de movimiento piro eléctrico HC-SR501, y posteriormente realizar una comunicación con el servidor ThingSpeak, el cual permitirá ver diferentes gráficas de los estados de los sensores, en intervalos de tiempo (cada 10 segundos), para finalmente realizar un análisis de la escena en la cocina, apuntando a las necesidades mencionadas anteriormente.

\section{MATERIALES Y MÉTODOS}

Esta sección presenta la información y las descripciones cortas de los procedimientos realizados para el análisis de la actividad que se desarrollara en una cocina tradicional, basados en el microcontrolador node mcu esp8266. Además, se hace una descripción de cada uno de los sensores, se muestra el diagrama de conexiones, posteriormente se muestra la etapa de diseño para que cada sensor tenga wifi. Por último, se realiza una conexión con el servidor ThingSpeak para el análisis por medio de graficas.

\section{Paso 1. Caracterización de los microcontroladores y sensores}

Para este proyecto se decidió trabajar en base al microcontrolador Node MCU ESP8266, ésta es una placa de desarrollo de bajo coste, de tamaño reducido, el cual tiene la gran ventaja de conectarse a internet, contiene 1 puerto analógico, 9 pines GPIO con I2C y SPI, cuenta adicionalmente con 4MB de memoria FLASH (32 Mbit) (BrikoGeek, 2019). En cuanto a sensores, se utilizó un sensor de flujo de agua; el objetivo es colocarlo en la boca de la llave del lavaplatos, y con la medición que haga éste, determinar si hay flujo de agua o no; en el mercado existen diferentes referencias de sensores de flujo de agua que se pueden conectar al Node, como lo son el YF-S401, con un tamaño de 1/4", con la capacidad de medir un caudal de 0,3 a 6L/min, y soportar una presión máxima de 0,8Mpa, las referencias FS300A con un tamaño de 3/4" y el FS4004 con un tamaño de 1", ambos con la capacidad de medir un caudal entre 1 a 60L/min y soportar una presión máxima de 1,2Mpa, y por último el YF-S201 con un tamaño de 1/2", tiene capacidad de medir de 1 a 30L/min y soportar una presión máxima de 1,75Mpa, (Naylampmechatronics, 2016). Analizando los datos anteriores se decidió elegir el YF-S201 dado que es el que soporta más presión y su tamaño es estándar.

Así mismo se consideró manejar un sensor magnético de puerta o ventana, el objetivo de éste es ser colocado en cada una de las gavetas de la cocina. Con la medición, el sensor dirá si está abierta o cerrada; se buscó uno que fuera pequeño, económico y que se pudiera conectar al node, se encontraron dos opciones, el T18AB, el cual al estar en presencia de un imán, el contacto que estaba cerrado se abre, (tdrobotica, 2011), pero este sensor no viene empaquetado, por lo cual su instalación se dificulta; el otro tipo de sensor magnético es el MC-38, el cual tiene una distancia de actuación de $15-25 \mathrm{~mm}$, viene recubierto de un material ABS, y la posibilidad de adaptarse con tornillos o cinta doble cara (Electronicapty, 2017); por lo mencionado anteriormente se eligió el MC-38. Por otro lado se eligió un sensor de corriente ACS712, el objetivo de este sensor, es ser puesto entre una tomacorriente y el conector del electrodoméstico, en este caso una licuadora, pero podría ser una olla arrocera, una batidora, un electrodoméstico que, al momento de encenderse, genere un cambio en el consumo de la corriente, entonces con la medición que realice el sensor, se podrá determinar si éste está encendido o apagado. Existen varias versiones de este sensor, las cuales son de $5 \mathrm{~A}$, con una sensibilidad de $185 \mathrm{mV} / \mathrm{A}$ y resolución de $26 \mathrm{~mA}$, la versión de $20 \mathrm{~A}$, con una sensibilidad de $100 \mathrm{mV} / \mathrm{A}$ y resolución de $49 \mathrm{~mA}$, y la versión $30 \mathrm{~A}$, con una sensibilidad de $66 \mathrm{mV} / \mathrm{A}$ y resolución de $74 \mathrm{~mA}$ (Naylampmechatronics, 2016). Se escogió este último dado que era el que tenía mejores características, en cuanto amperaje, sensibilidad y resolución.

Por último, se incluyó un sensor de movimiento con el objetivo de ser puesto en la entrada de la cocina para registrar cuando se ingresa a ella o no. La medición de éste a través del sistema anteriormente descrito ayuda a detectar el movimiento; actualmente se pueden encontrar diferentes tipos como el sensor de movimiento infrarrojo de keyestudio, con ángulo de detección $100^{\circ}$, distancia de detección 7 metros (Innovadidactic, 2019); Sensor de Movimiento PIR Parallax 555, el cual detecta una persona hasta 30 pies de distancia ( 9,14 metros), o hasta 15 pies de distancia en modo de sensibilidad reducido (Parallax, 2019), el sensor de movimiento Infrarrojo Pir mini HC-SR 505, el cual tiene una distancia de detección de 3 metros y 100 grados en un ángulo de cono (Moviltronics, 2019). Igualmente, el sensor HC-SR501 el cual puede detectar movimiento de 3 a 7 metros en un ángulo de 100 grados, ángulos en forma de cono (Moviltronics, 2019); exceptuando el sensor mini HC-SR 505 todos los sensores de movimiento tienen las mismas prestaciones, pero se escogió el HC-SR501 por ser el más económico y es comercializado en la ciudad. 
Los sensores elegidos anteriormente tienen las siguientes características:

Sensor de flujo de agua YF-S201: Éste el efecto hall localizado en la parte externa, el cual detecta el campo magnético de las paletas, las cuales tienen un imán y están en la parte interna del rotor aislado para evitar fugas de agua. Este sensor de efecto hall envía los pulsos al microcontrolador, los cuales serán convertidos en flujo (Naylampmechatronics, 2016).

Sensor de corriente ACS712: Es un dispositivo que mide la variación del campo magnético de una sola línea, en la cual se produce una inducción cuando la corriente pasa por éste y la calcula internamente con la ayuda de un sensor de efecto hall (Naylampmechatronics, 2016).

Sensor magnético de puerta MC-38: Este sensor es utilizado comúnmente en puertas, ventanas, tiene un tipo de contacto que es para ( $\mathrm{N} / \mathrm{C}$ ) circuitos normalmente cerrados, lo que significa que cuando los imanes se alinean, se cierra el interruptor (Ardobot, 2018).

Sensor de movimiento Piro eléctrico HC-SR501: Éste contiene posee un piezo eléctrico que detecta la radiación emitida por cualquier objeto y la transforma en una señal eléctrica, cada sensor está dividido en dos campos; cuando miden las diferencias del infrarrojo, resulta una señal eléctrica, la cual emite una señal digital que dispara una alarma. Por el contrario, si la cantidad de infrarrojo es la misma, la señal eléctrica resultante es nula. Este sensor divide el espacio en zonas con la ayuda de lentes que se encuentran en el semicírculo de plástico, los cuales ayudan a enfocar la radiación (Llamas, 2015).

\section{Paso 2. Conexiones eléctricas}

En esta primera etapa se consultó los diagramas eléctricos correspondientes a cada uno de los sensores mencionados anteriormente, después de esto, se creó dentro de PROTEUS tanto el node y los pines de sensores para mostrar así de manera más sencilla las conexiones, como se muestra en las figuras 1 y 2.

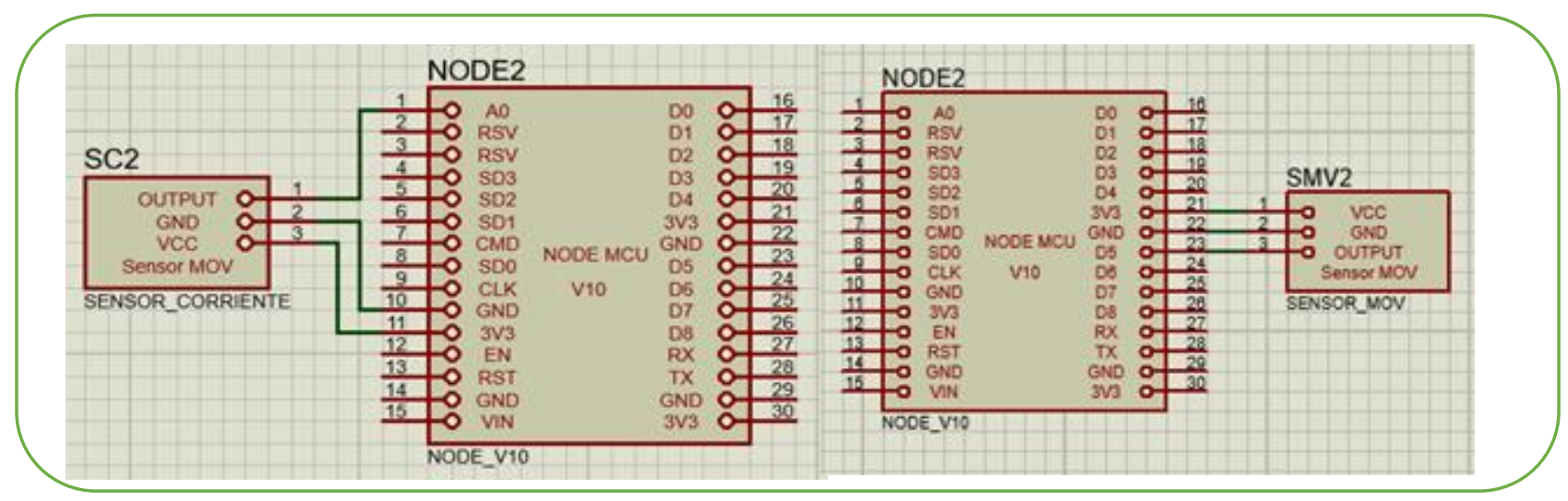

Fig. 1: Conexiones eléctricas sensor de corriente (izquierda) y sensor de movimiento (derecha).

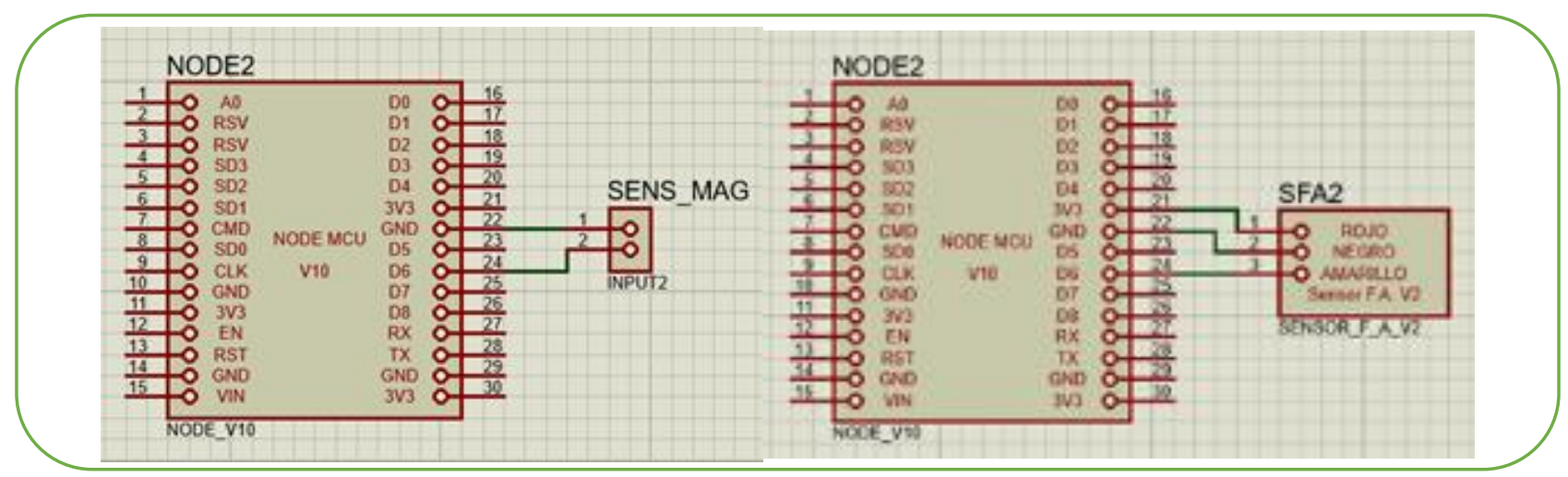

Fig. 2: Conexiones sensores sensor magnético (izquierda) y sensor de flujo de agua (derecha) 
En la tabla 1 se puede observar un resumen de las conexiones mostradas en la figura 1 y 2.

Tabla 1: Conexiones Sensor magnético de puerta MC-38, Sensor de corriente ACS712, Sensor de Flujo YF-S201 y Sensor de movimiento HC-SR501.

\begin{tabular}{|l|l|}
\hline \multicolumn{1}{|c|}{ Sensor magnético de puerta MC-38 } & Node Mcu Esp8266 \\
\hline Pin 1 & D6 \\
\hline Pin 2 & GND \\
\hline Sensor de corriente ACS712 & Node Mcu Esp8266 \\
\hline VCC & $3.3 V$ \\
\hline OUT & A0 \\
\hline GND & GND \\
\hline Sensor de flujo de agua YF-S201 & Node Mcu Esp8266 \\
\hline Pin Rojo & $3.3 V$ \\
\hline Pin Amarillo & D6 \\
\hline Pin negro & GND \\
\hline Sensor de movimiento Piro eléctrico HC-SR501 & Node Mcu Esp8266 \\
\hline VCC & $3.3 V$ \\
\hline OUT & D5 \\
\hline GND & GND \\
\hline
\end{tabular}

\section{Paso 3. Programación de sensores}

Se realizó una programación en el lenguaje $\mathrm{C}_{++}$, por medio de la interfaz IDE-ARDUINO, en el microcontrolador Node MCU ESP8266 para la lectura de cada sensor y se calibró de acuerdo con las necesidades de cada sensor, a continuación se muestra una breve explicación de la programación realizada a cada uno de los sensores:

Sensor de flujo de agua YF-S201: Primero se inicializaron las variables globales NUMPULSOS, PINSENSOR=2,FACTOR CONVERSION=7,5 dado que el diámetro es 1/2 " (Naylampmechatronics, 2018), Segundo, en el VOID SETUP se inicializó la comunicación, luego se configuró PINSENSOR=ENTRADA. Después de esto se configuró la función de interrupción, tercero en el VOID LOOP se obtuvo LA FRECUENCIA, EL CAUDAL_L_M y el CAUDAL_L_H, se realizó un condicional de tipo IF donde se preguntó si el CAUDAL_L_M era igual a cero, si era VERDADERO se imprimía el valor del caudal y el mensaje "llave cerrada" y si era FALSO se imprimía el valor del cauda y el mensaje "llave abierta", como se observa en el diagrama de Flujo de la tabla 2, literal A.

Sensor de corriente ACS712: Primero se inicializaron las variables globales SENSIBILIDAD $=0,66$, esta sensibilidad es debido al modelo del sensor de corriente que puede medir hasta $30 \mathrm{~A}$, y se declaró una variable OFFSET=0; que se utilizó posteriormente en otros cálculos. Segundo, en el VOID SETUP se inicializó la comunicación. Tercero, en el VOID LOOP se obtuvo la corriente IP, la corriente IRMS, y la POTENCIA, luego se realizó un condicional de tipo IF donde se preguntó si el valor de la POTENCIA era mayor a 13, dado que en la calibración se evidenció que cuando la licuadora se encendía, se obtenía una potencia superior a 13, si era VERDADERO se imprimía un mensaje por el monitor serial el valor de la POTENCIA y un mensaje de "licuadora encendida" y si era FALSO se imprimía el valor de la potencia y un mensaje "licuadora apagada", como se observa en el diagrama de flujo de la tabla 2, literal B. 
Sensor magnético de puerta MC-38 (Tabla 3): Primero se inicializaron las variables globales PIN_INT=3 y Estado=LOW. Segundo, en el VOID SETUP se inició la comunicación y se determinó que PIN_INT=INPUT_PULLUP, luego se configuró la función de interrupción la cual realizó el llamado a la función de CAMBIOS. Tercero, en el VOID LOOP se realizó un condicional de tipo IF donde se preguntó si el Estado era HIGH, si era VERDADERO se imprimía un mensaje por el monitor serie "Gaveta abierta" y si era FALSO se imprimía un mensaje "Gaveta cerrada", tal y como se observa en el diagrama de bloques parte a.

Sensor de movimiento Piro eléctrico HC-SR501: Primero se inicializaron las variables globales PIRPIN=4; y VALUE $=0$. Segundo, en el VOID SETUP se inicializó la comunicación y se configuró PIRPIN=INPUT. Tercero, en el VOID LOOP se realizó un condicional tipo IF donde se preguntó si VALUE=HIGH, si era VERDADERO se imprimía "Sí hay movimiento" o por el contrario FALSO se imprimía "No hay movimiento" tal y como se observa en el diagrama de bloques de la tabla 3 parte $\mathrm{B}$.

Tabla 2: Diagramas de flujo sensor de flujo de agua YF-S201 y Sensor de corriente ACS712.

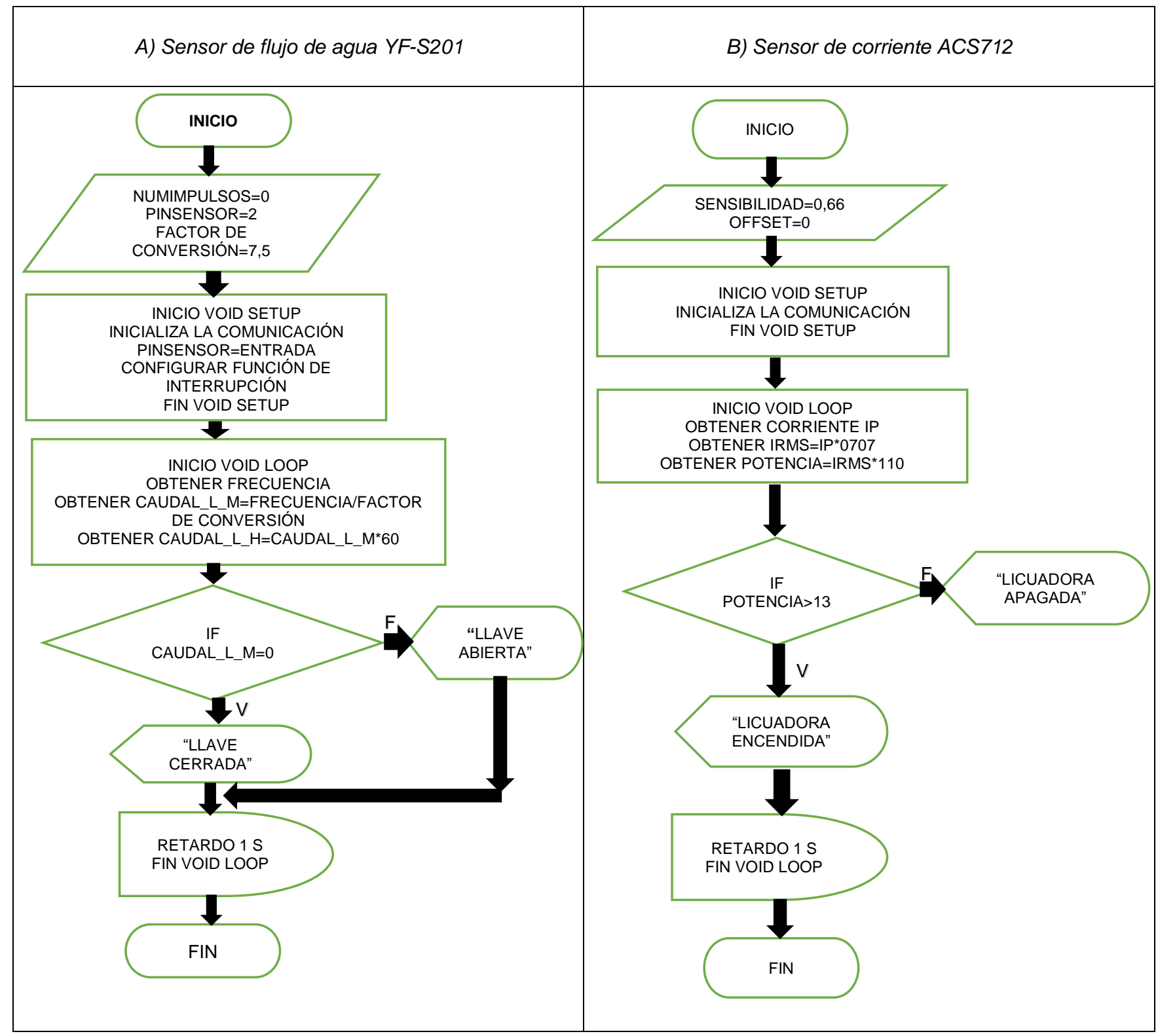


Tabla 3: Diagramas de flujo Sensor magnético de puerta MC-38 y Sensor de movimiento Piro eléctrico HC-SR501.

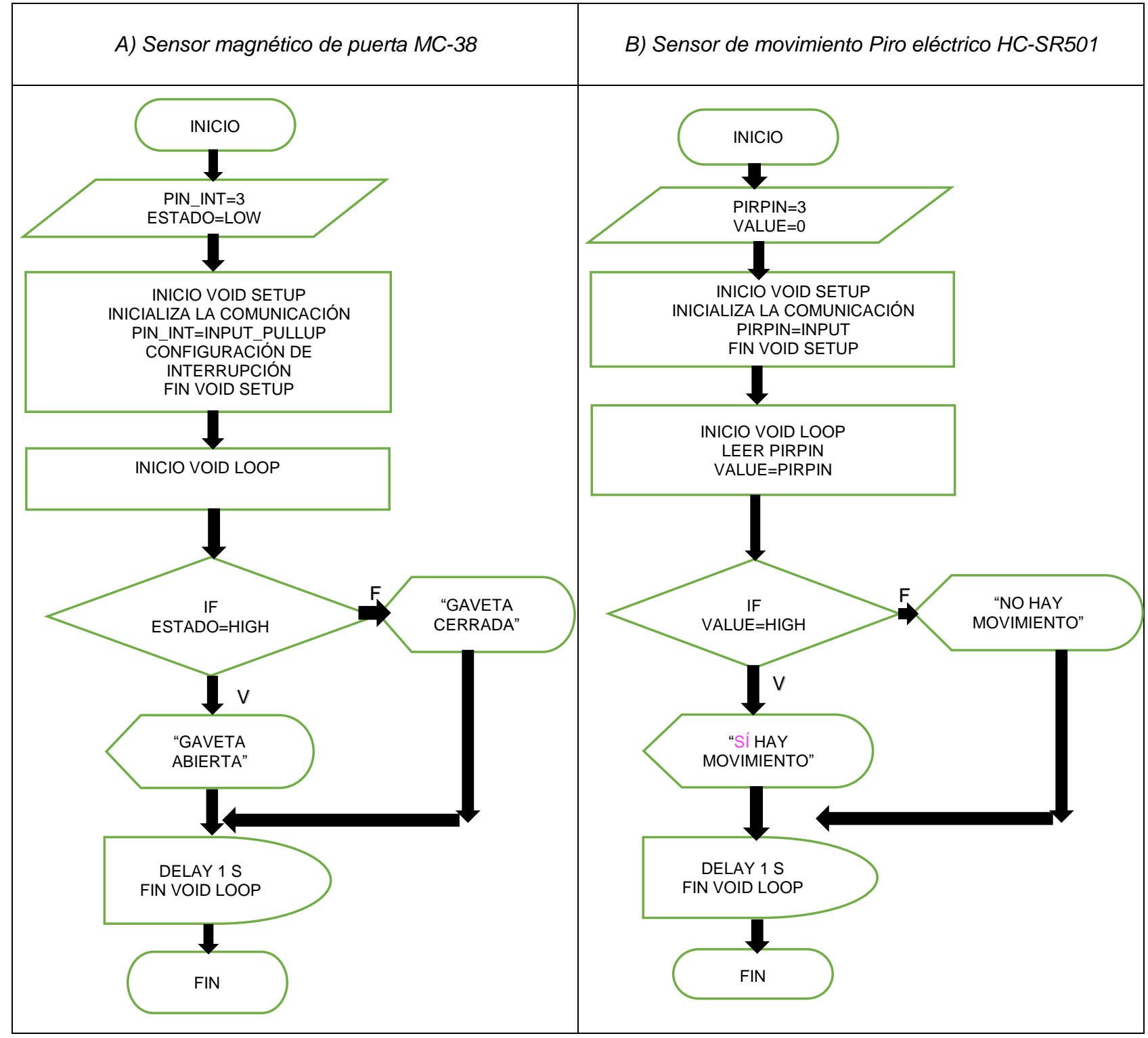

\section{Paso 4. Diseño Placa-Fase Alimentación}

Para la alimentación autónoma del node, se implementó una entrada INPUT1 la cual se va a alimentar con una batería de 9V, después se añadió un SWITCH ON/OFF para tener el control en el encendido y apagado de la PCB, posteriormente se ejecutó la etapa de rectificación, que viene dada por un diodo 1N4004, 2 condensadores de $22 \mathrm{uF}$ y $100 \mathrm{nF}$ respectivamente, y el regulador a 5V LM7805, y en la salida OUTPUT se adaptó un cable con punta MicroSD para alimentar el node, como se observa en la figura 3.

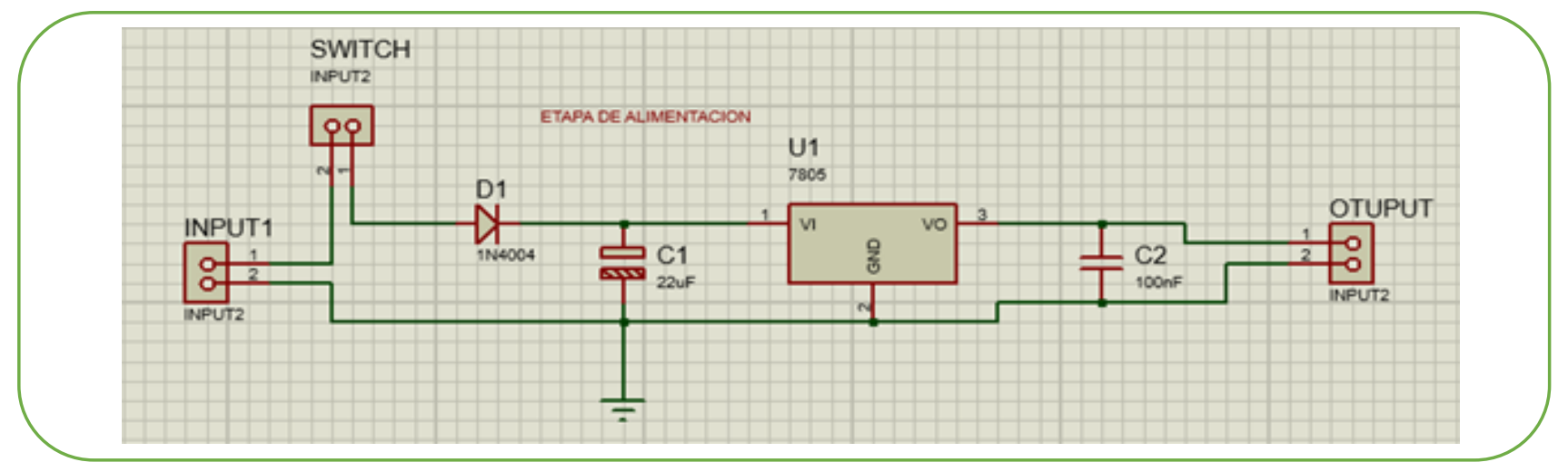

Fig. 3: Etapa de Alimentación 


\section{Paso 5. Diseño Placa-Fase Visualización}

Para poder visualizar el estado de cada sensor, se agregó al node un conversor I2C y una pantalla LCD de 16x2, el esquema de la conexión de estos con el node es el que se observa en la figura 4. La conexión entre el conversor I2C y la pantalla es paralela, el conversor I2C contiene tiene 4 pines, los cuales se muestran en el esquemático como PART_SUP_CONV que van al node de la siguiente forma, pin VCC $\rightarrow$ Vin, pin GND $\rightarrow$ GND, pin SDA $\rightarrow$ D2, SDL $\rightarrow$ D1.

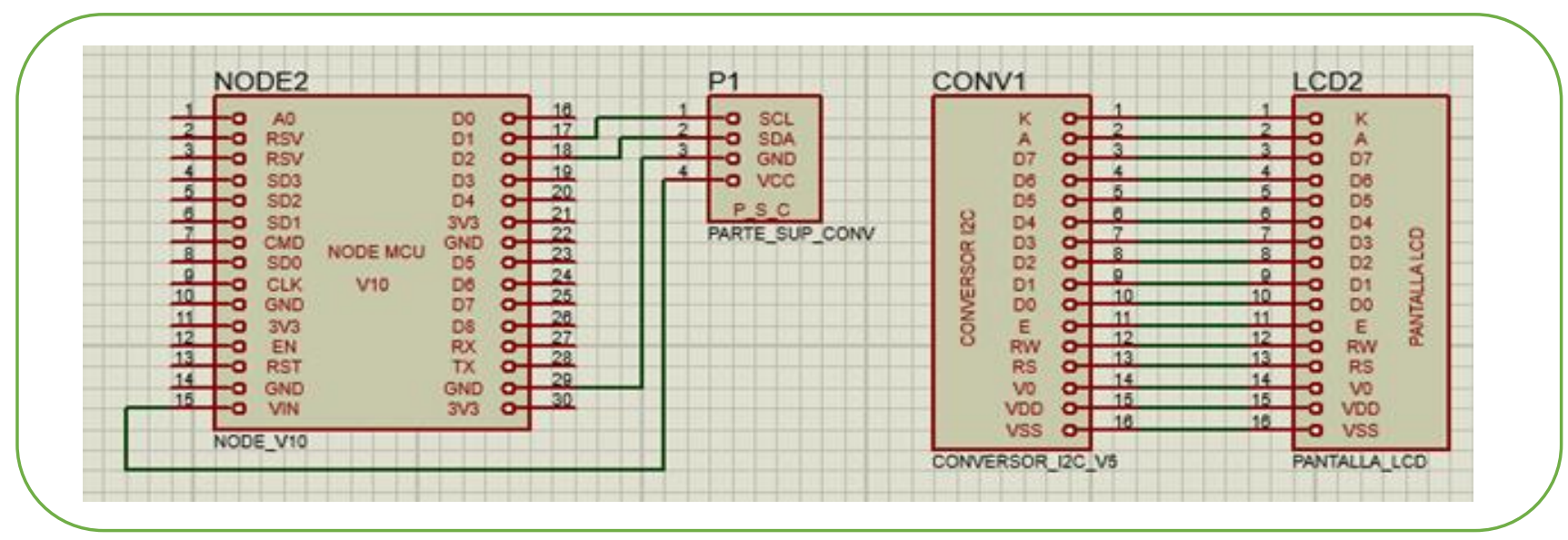

Fig. 4: Etapa de visualización.

Adicionalmente, la visualización que se observa a través de la pantalla LCD tendrá la siguiente codificación, como se observa en la tabla 4, dado que el espacio de la pantalla es pequeño y no se pueden publicar mensajes extensos, como se observaba por el monitor serie.

Tabla 4: Codificación sensores

\begin{tabular}{|c|c|}
\hline \multicolumn{2}{|c|}{ Codificación } \\
\hline Carácter & Significado \\
\hline G1 & Gaveta 1 abierta \\
\hline G0 & Gaveta 1 cerrada \\
\hline L1 & Licuadora encendida \\
\hline L0 & Licuadora apagada \\
\hline M1 & Sí hay movimiento en la cocina \\
\hline M0 & No hay movimiento en la cocina \\
\hline LV1 & Lavaplatos abierto \\
\hline LV0 & Lavaplatos cerrado \\
\hline
\end{tabular}

\section{Paso 6. Creación Layaut y pistas}

Se realizaron todas las conexiones mencionadas con anterioridad a cada uno de los sensores, y se obtuvo el layaut y las pistas para la impresión, como se observa en las figuras 5,6,7 y 8 .

Para el sensor de flujo de agua:

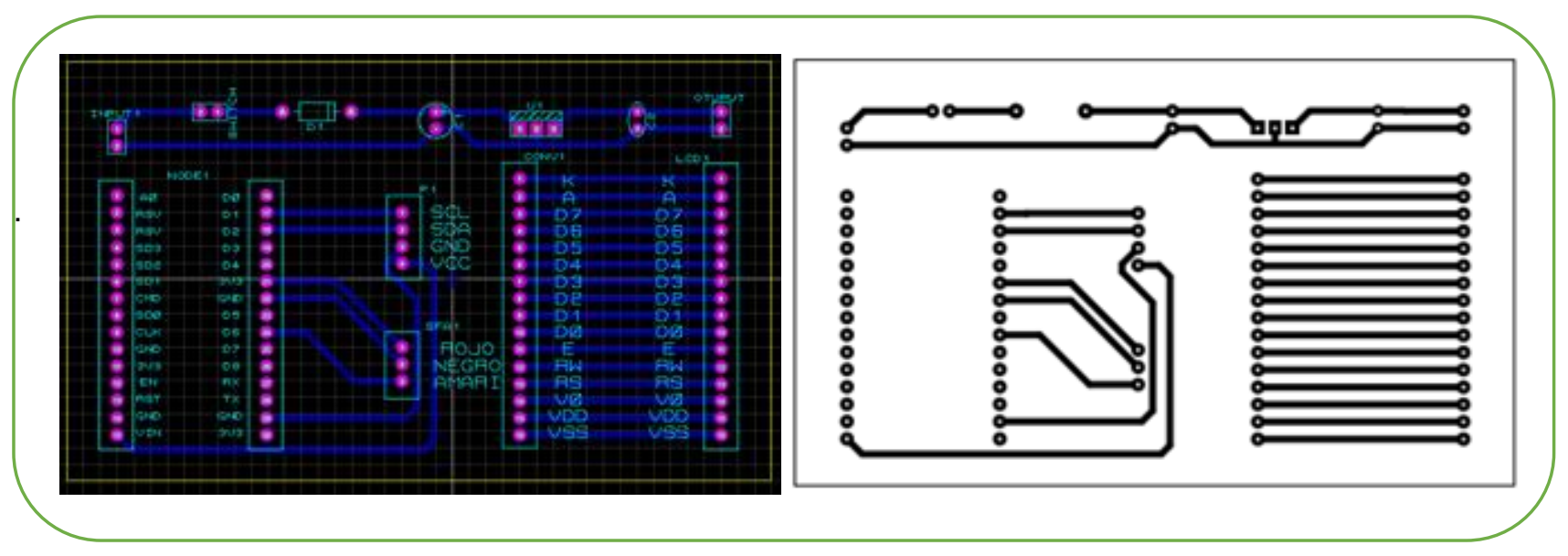

Fig. 5: Sensor de flujo de agua Layaut (Izquierda), pistas (Derecha). 
Para el sensor de magnético de puerta MC-38.

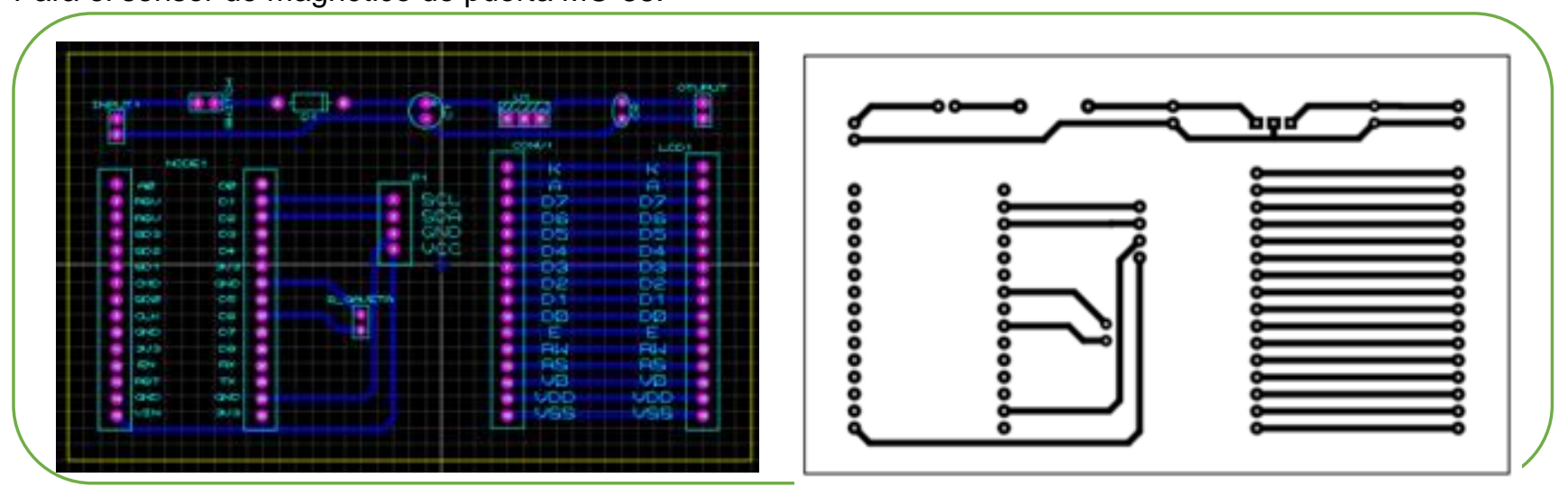

Fig. 6: Sensor magnético Layaut (Izquierda), pistas (Derecha).

Para el sensor magnético de movimiento.
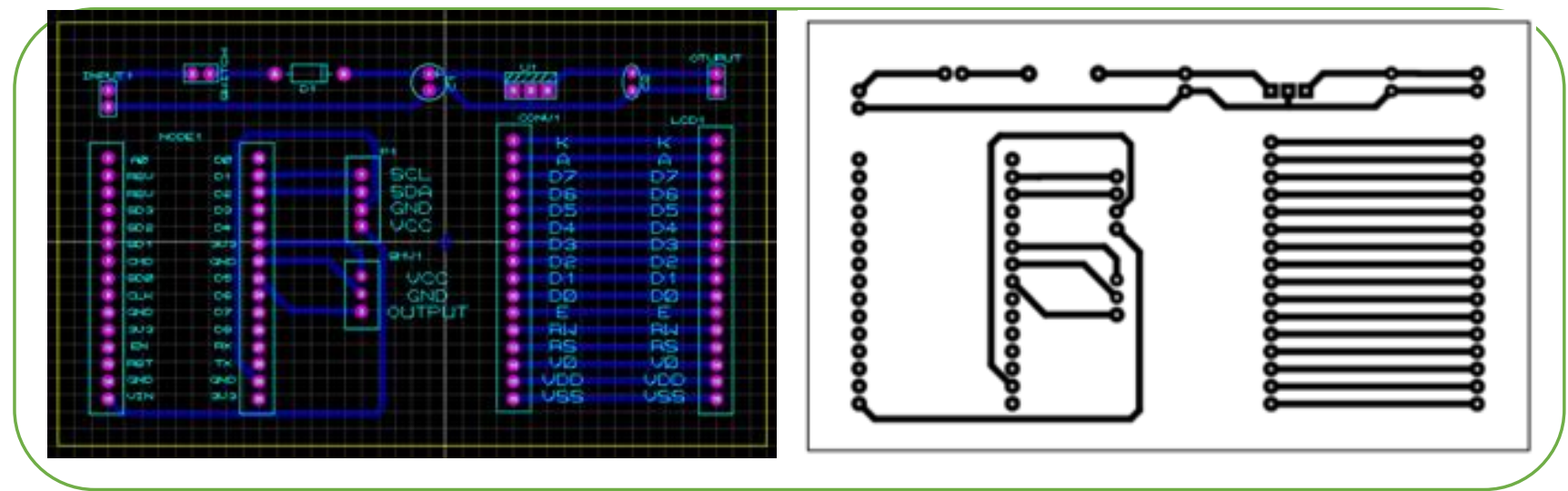

Fig. 7: Sensor de movimiento Layaut (Izquierda), pistas (Derecha).

Para el sensor magnético de corriente:

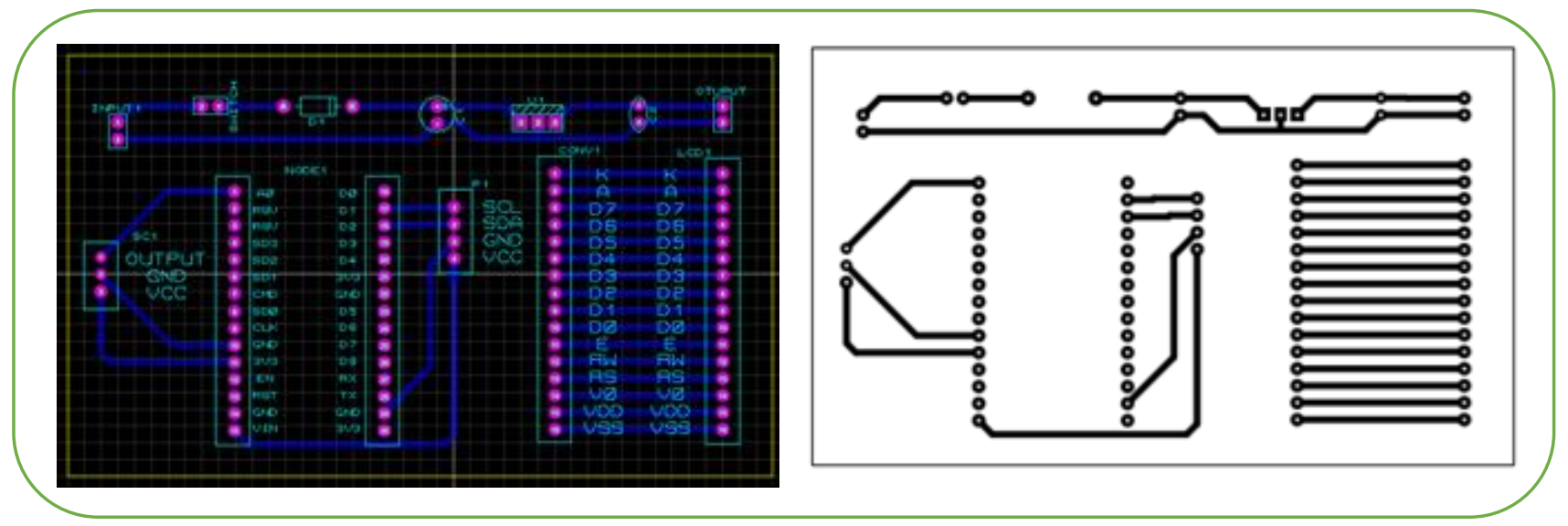

Fig. 8: Sensor de corriente Layaut (Izquierda), pistas (Derecha).

\section{Paso 7. Conexión al servidor gratuito ThingSpeak}

ThingSpeak es una herramienta que se puede usar de manera gratuita, y sirve para enviar datos del sensor de forma privada a la nube, compartir datos en canales públicos, se pueden analizar y visualizar los datos con Matlab, se pueden generar alarmas, programar eventos, y funciona con microcontroladores de uso común como lo son: Arduino, Raspberry, ESP8266 modulo wifi y Node Mcu. Para este proyecto se creó un canal de uso personal llamado SENSORES EN LA COCINA y 4 campos llamados respectivamente, sensor de corriente, sensor de movimiento, sensor de flujo de agua y sensor magnético de puerta, por lo tanto, cada uno 
de los sensores se comunicará con cada campo. La aplicación ThingSpeak genera 2 claves API y una identificación ID, las cuales van especificadas en el código para poder lograr la comunicación.

\section{RESULTADOS}

Primero se describen los resultados obtenidos en la programación individual de cada uno de los sensores por medio de la vista del monitor serial, segundo se muestra la construcción de los PCB que corresponde a su fase de alimentación, visualización, posteriormente la comunicación con el servidor ThingSpeak, y el análisis por medio de gráficas de los estados de los sensores.

\section{Pasos 1,2 y 3. Caracterización, Conexiones eléctricas, programación de sensores}

Se muestran los resultados obtenidos después de realizar las conexiones señaladas en la tabla 1 y las programaciones que se muestran en diagramas de flujos en las tablas 2 y 3 , adicionalmente se modificó el programa original para realizar pruebas de acierto, a cada sensor se le destinó una letra aleatoria, para que al momento de digitarle en el monitor serie, nos arrojara el resultado respectivo, como se describe a continuación.

Sensor magnético de puerta MC-38: Se realizó el montaje en una gaveta tradicional, la distancia de activación es aproximadamente entre $15-25 \mathrm{~mm}$, se abrió la gaveta y se obtuvo el mensaje de abierto, se cerró la gaveta y arrojó el mensaje de cerrado, tal y como se planteó en el diagrama de bloques y como se observa en la figura 9. Como se mencionó anteriormente, se realizó una modificación al programa básico para que al presionar la letra "G" (Gaveta) el programa arrojara el estado del sensor, se realizaron 3 intentos para cada uno de los estados (cerrado o abierto) en cada uno de los cuales se presionaba la letra "G" 20 veces para solicitar la información, obteniendo así los resultados de la tabla 5.

Sensor de corriente ACS712: Se realizaron dos pruebas, una sin adaptarle ningún electrodoméstico, el cual arrojó el valor de Ip e Irms cercanos a cero y una potencia aproximada de 1,6W, al momento de adaptarle una licuadora tradicional, se encontró que conectándola sin encenderla valores Ip, Irms y potencia cercanos cero, cuando se encendió la licuadora se observó un aumento en la potencia hasta de 50W y que se estabilizo en aproximadamente en $15.3 \mathrm{~W}$, por lo tanto en la programación se tomó $13 \mathrm{~W}$ para determinar si la licuadora estaba encendida o apagada, tal y como se planteó en el diagrama de flujo y como se muestra en la figura 10. De igual manera se realizó una modificación al programa básico para que al presionar la letra "C" (Corriente) el programa arrojara el estado del sensor; se realizaron 3 intentos para cada uno de los estados (licuadora encendida o licuadora apagada) en cada uno de los cuales se presionaba la letra "C" 20 veces, obteniendo así los resultados de la tabla 6.

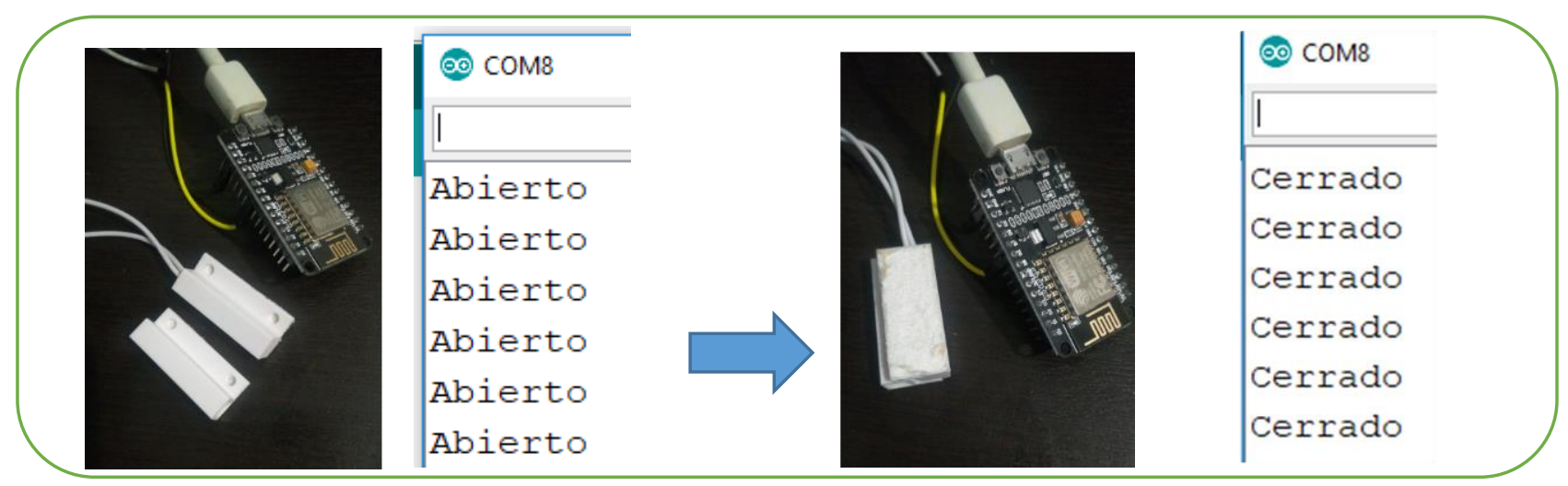

Fig. 9: Resultados sensor magnético de puerta MC-38 - Abierto y Cerrado.

Tabla 5: Sensor magnético de puerta MC-38-Prueba de acierto.

\begin{tabular}{|l|l|l|l|}
\hline \multicolumn{4}{|c|}{ Sensor magnético de puerta mc-38 estado abierto } \\
\hline Pruebas & Intentos & Aciertos & Porcentaje de efectividad \\
\hline 1 & 20 & 20 & $100 \%$ \\
\hline 2 & 20 & 20 & $100 \%$ \\
\hline 3 & 20 & 20 & $100 \%$ \\
\hline \multicolumn{4}{|c|}{ Sensor magnético de puerta mc-38 estado cerrado } \\
\hline Pruebas & Intentos & Aciertos & Porcentaje de efectividad \\
\hline 1 & 20 & 20 & $100 \%$ \\
\hline 2 & 20 & 20 & $100 \%$ \\
\hline 3 & 20 & 20 & $100 \%$ \\
\hline
\end{tabular}




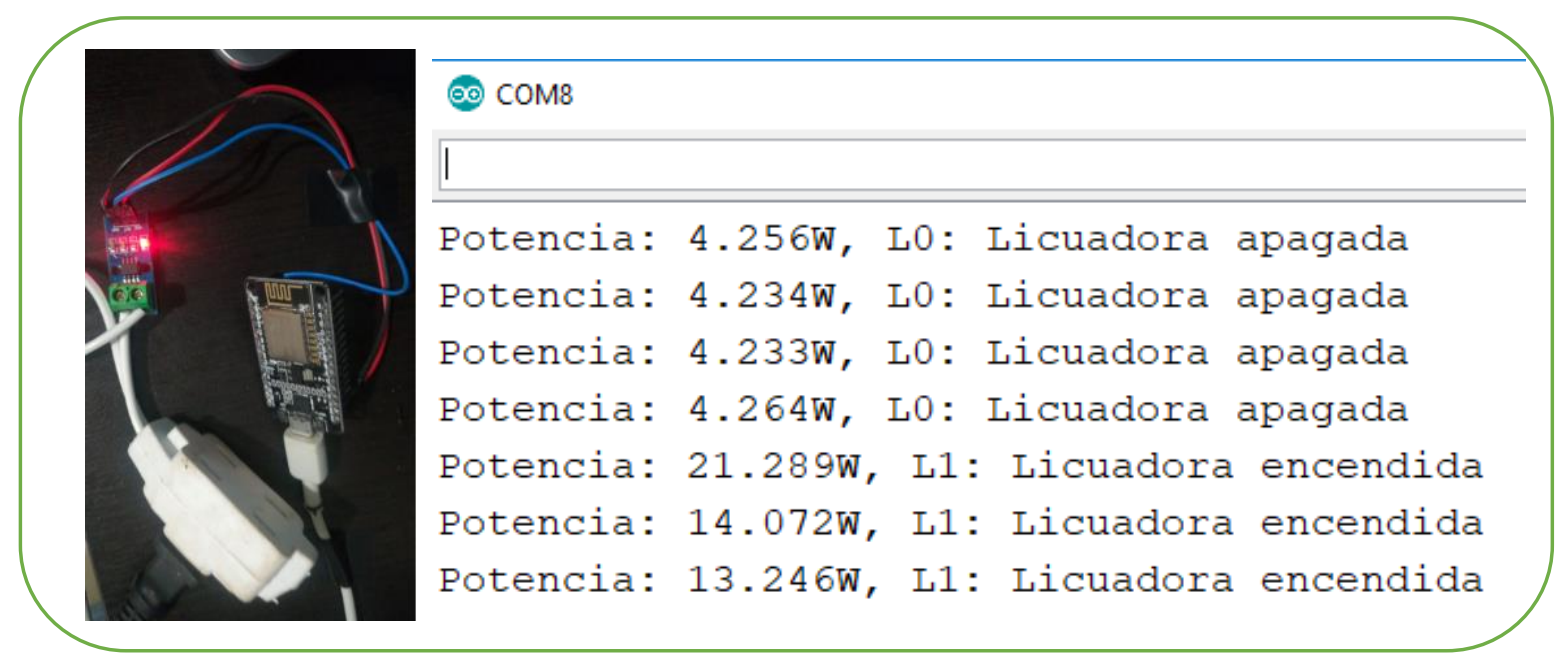

Fig. 10: Resultados sensor de corriente ACS712 con licuadora adaptada.

Tabla 6: Sensor de corriente ACS712-Prueba de aciertos.

\begin{tabular}{|c|c|c|c|}
\hline \multicolumn{3}{|c|}{ Sensor de corriente acs712-licuadora apagada } \\
\hline Pruebas & Intentos & Aciertos & Porcentaje de efectividad \\
\hline 1 & 20 & 20 & $100 \%$ \\
\hline 2 & 20 & 20 & $100 \%$ \\
\hline 3 & 20 & 20 & $100 \%$ \\
\hline \multicolumn{4}{|l|}{ Sensor de corriente acs712-licuadora encendida } \\
\hline Pruebas & Intentos & Aciertos & Porcentaje de efectividad \\
\hline 2 & 20 & 20 & $100 \%$ \\
\hline 3 & 20 & 20 & $100 \%$ \\
\hline
\end{tabular}

Sensor de flujo de agua YF-S201: Se adaptó el sensor a una llave del lavaplatos de manera superficial cuando la llave está cerrada, muestra un caudal de 0.00L/min y un mensaje de "llave cerrada", como se planteó en los diagramas de flujo y como se observa en la sección a) de la figura 3. Cuando se abrió la llave, de igual manera se mostró el valor del caudal y el respectivo mensaje de "llave abierta", como se observa en la sección b) de la figura 11. Así mismo, se realizó una modificación al programa básico para que al presionar la letra "F" (Flujo de agua) el programa arrojara el estado del sensor, se realizaron 3 intentos para cada uno de los estados (llave abierta o llave cerrada) en cada uno de los cuales se presionaba la letra "F" 20 veces, obteniendo así los resultados de la tabla 7.
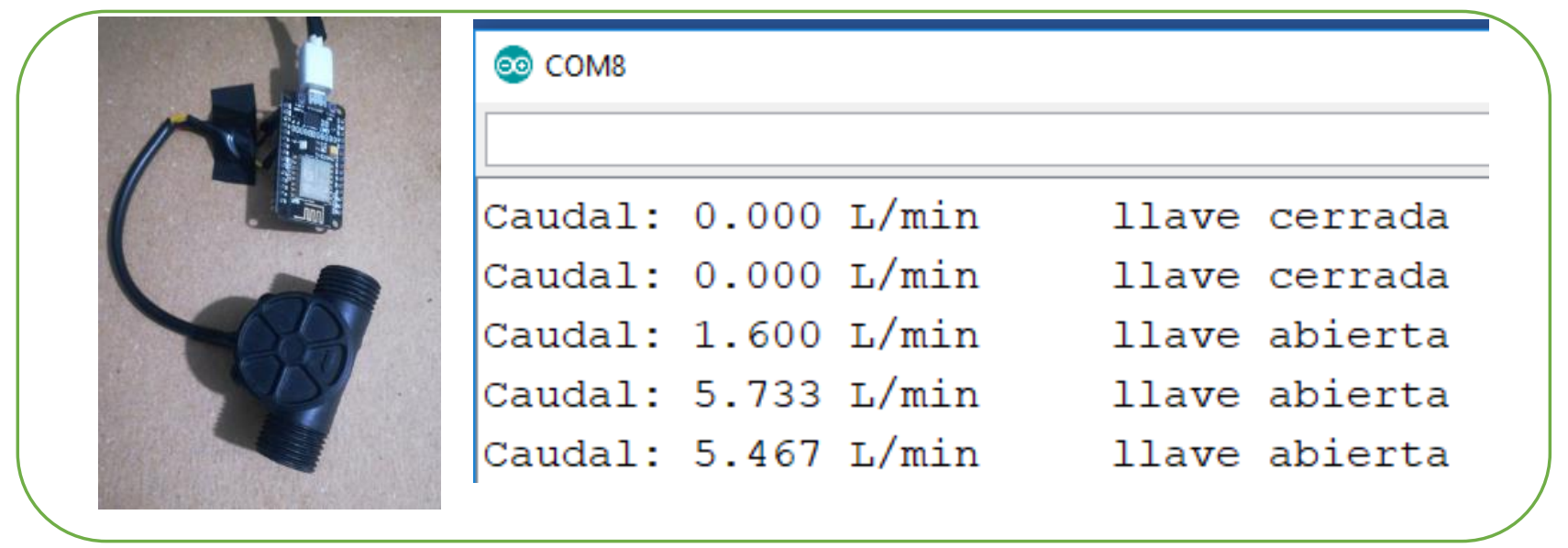

Fig. 11: Resultados para el sensor de flujo YF-S201

Es de anotar que L/m significa los Litros por Minuto. 
Tabla 7: Sensor de flujo de agua YF-S201-Prueba de aciertos

\begin{tabular}{|c|c|c|c|}
\hline \multicolumn{4}{|c|}{ Sensor flujo de agua YF-S201- Ilave cerrada } \\
\hline Pruebas & Intentos & Aciertos & Porcentaje de efectividad \\
\hline 1 & 20 & 20 & $100 \%$ \\
\hline 2 & 20 & 20 & $100 \%$ \\
\hline 3 & 20 & 20 & $100 \%$ \\
\hline \multicolumn{3}{|c|}{ Sensor flujo de agua YF-S201- Ilave abierta } \\
\hline Pruebas & Intentos & Aciertos & Porcentaje de efectividad \\
\hline 1 & 20 & 20 & $100 \%$ \\
\hline 2 & 20 & 20 & $100 \%$ \\
\hline 3 & 20 & $100 \%$ \\
\hline
\end{tabular}

Sensor de movimiento Piro eléctrico HC-SR501: Se colocó el sensor sobre una superficie de tal manera que se pudieron realizar movimientos con las manos enfrente de él; cuando se movió la mano, arrojó el mensaje de señal activa y en caso contrario señal inactiva, tal cual se planteó en los diagramas de bloque, y se muestra en la figura 12. De igual manera se realizó una modificación al programa básico para que al presionar la letra "M" (Movimiento) el programa arrojara el estado del sensor, se realizaron 3 intentos para cada uno de los estados (hay movimiento en la cocina, o no hay movimiento en la cocina) en cada uno de los cuales se presionaba la letra "M" 20 veces, obteniendo así los resultados de la tabla 8.

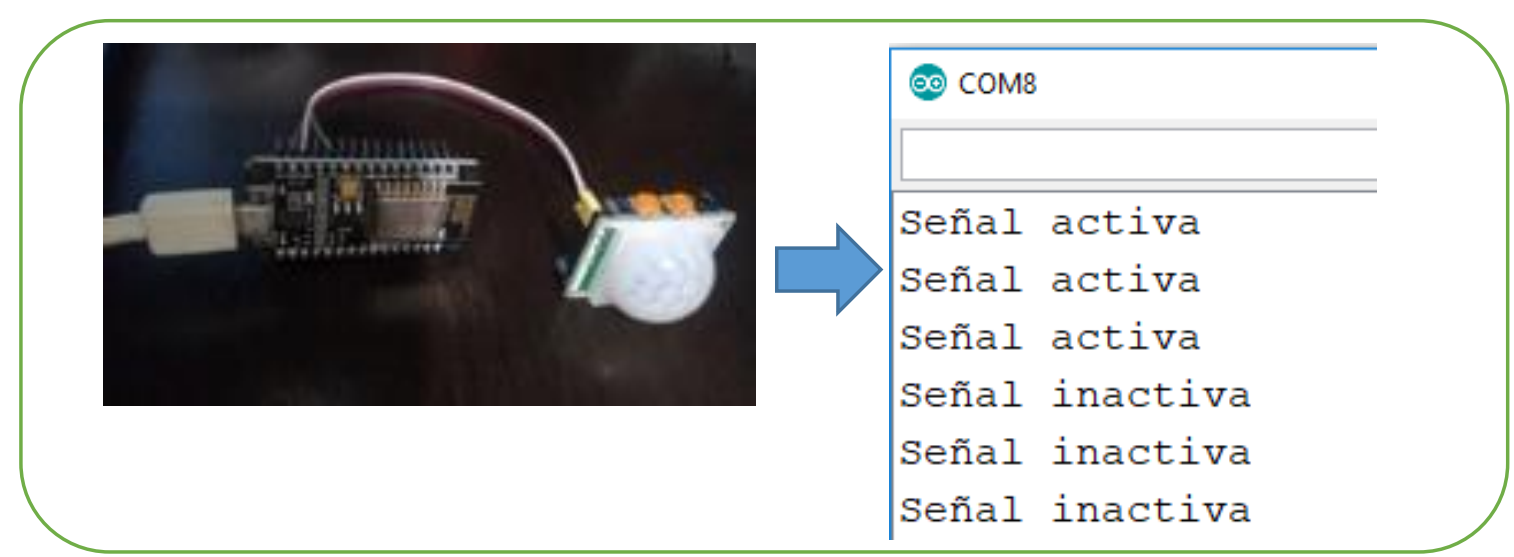

Fig. 12: Resultados sensor de movimiento piroelectrico HC-SR501- Señal Inactiva y activa.

Tabla 8: Sensor de movimiento piro eléctrico HC-SR501-Prueba de aciertos.

\begin{tabular}{|c|c|c|c|}
\hline \multicolumn{3}{|c|}{ Sensor de movimiento piroelectrico hc-sr501 - Sin movimiento } \\
\hline Pruebas & Aciertos & $\begin{array}{c}\text { Porcentaje } \\
\text { efectividad }\end{array}$ \\
\hline 1 & 20 & 20 & $100 \%$ \\
\hline 2 & 20 & 20 & $100 \%$ \\
\hline 3 & 20 & 20 & $100 \%$ \\
\hline \multicolumn{2}{|c|}{ Sententos } & Aciertos & $\begin{array}{c}\text { Porcentaje de } \\
\text { efectividad }\end{array}$ \\
\hline Pruebas & Intentos & 16 & $80 \%$ \\
\hline 1 & 20 & 18 & $90 \%$ \\
\hline 2 & 20 & 19 & $95 \%$ \\
\hline 3 & 20 & & \multicolumn{2}{c|}{} \\
\hline
\end{tabular}

Dado que cuando se realiza el análisis con movimiento hay una variación, se realizó una prueba de varianza en la cual se realizaron 20 intentos para cada réplica, y para cada tratamiento, como se observa en la tabla 9.

Tabla 9: Datos análisis varianza.

\begin{tabular}{|c|c|c|c|c|c|c|c|c|c|c|c|}
\hline T Proceso & \multicolumn{7}{|c|}{ Réplicas } & Sum \\
\hline Sen Mov & 1 & 2 & 3 & 4 & 5 & 6 & 7 & 8 & 9 & 10 & \\
\hline Tratam 1 & 80 & 95 & 90 & 75 & 80 & 85 & 95 & 80 & 80 & 85 & 845 \\
\hline Tratam 2 & 85 & 80 & 80 & 90 & 95 & 90 & 90 & 95 & 90 & 90 & 885 \\
\hline Tratam 3 & 90 & 95 & 80 & 85 & 90 & 95 & 80 & 85 & 80 & 80 & 860 \\
\hline Tratam 4 & 95 & 90 & 80 & 85 & 80 & 80 & 90 & 95 & 80 & 80 & 855 \\
\hline
\end{tabular}


Luego, con un intervalo de confianza del 99,5\%, el sistema tiene una efectividad del $86,125 \%$ de las veces, obteniendo como resultado que EL MODELO NO ES SIGNIFICATIVO, lo cual indica que el sistema es estable, como se evidencia en la tabla 10.

Tabla 10: Resultado análisis de datos varianza

\begin{tabular}{|l|c|c|c|c|c|}
\hline Fuente Varia & G L & SC & CM & Fcal & F Tab \\
\hline Sum C Total & & 1474,38 & & & \\
\hline Tratamiento & 3 & 86,875 & 28,958 & 0,75 & 5,0616 \\
\hline Error Exper & 36 & 1387,5 & 38,542 & & \\
\hline Total & 39 & 1474,38 & 67,5 & & \\
\hline $\begin{array}{l}\text { El modelo no es } \\
\text { significativo }\end{array}$ & & & & & \\
\hline
\end{tabular}

Paso 4,5,6. Diseño Placa-Fase Alimentación, Diseño Placa-Fase Visualización, Creación Layaut y pistas

Se imprimieron las pistas creadas en el paso 6, por una cara, se realizó la conexión de la alimentación, la cual es una pila de $9 \mathrm{~V}$ alcalina, se conectaron un interruptor, y un adaptador que va de la salida de la etapa de alimentación a la entrada mini usb del node, se instaló el conversor i2c, la pantalla LCD 16X2 y por último se colocó el sensor correspondiente; al lado derecho de las figuras 13, 14, 15 y 16 se observa el estado de los sensores por medio de la pantalla LCD, con lo cual se nota el correcto funcionamiento del montaje.

Para el sensor de flujo de agua:

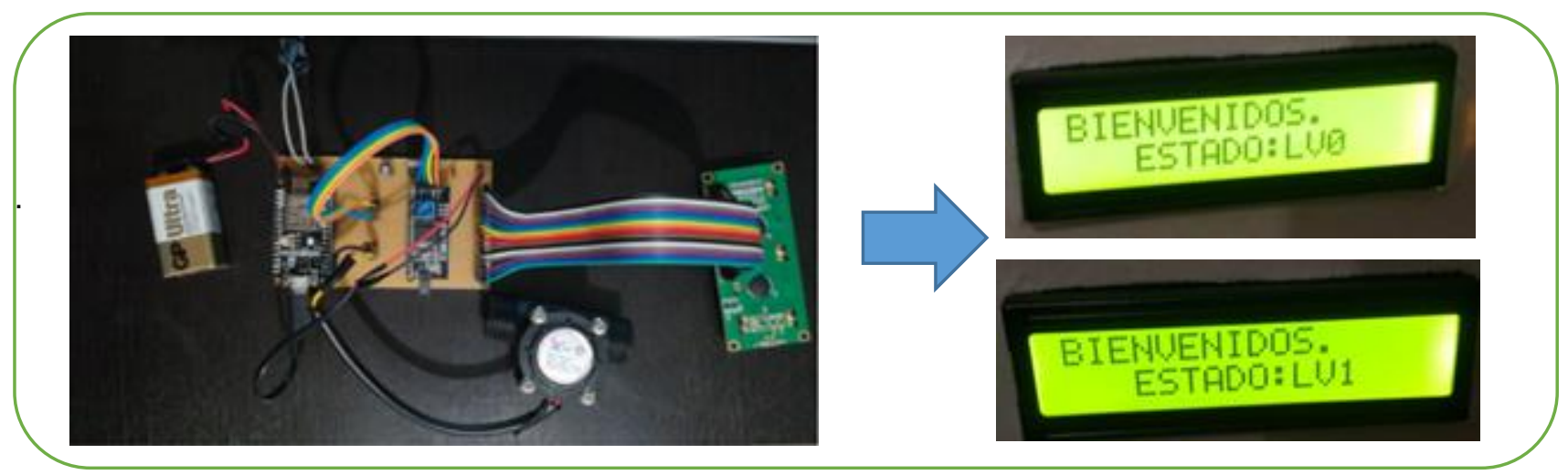

Fig. 13: Montaje PCB sensor de flujo de agua (Izquierda), Estados LCD(Derecha).

Para el sensor de magnético de puerta MC-38.

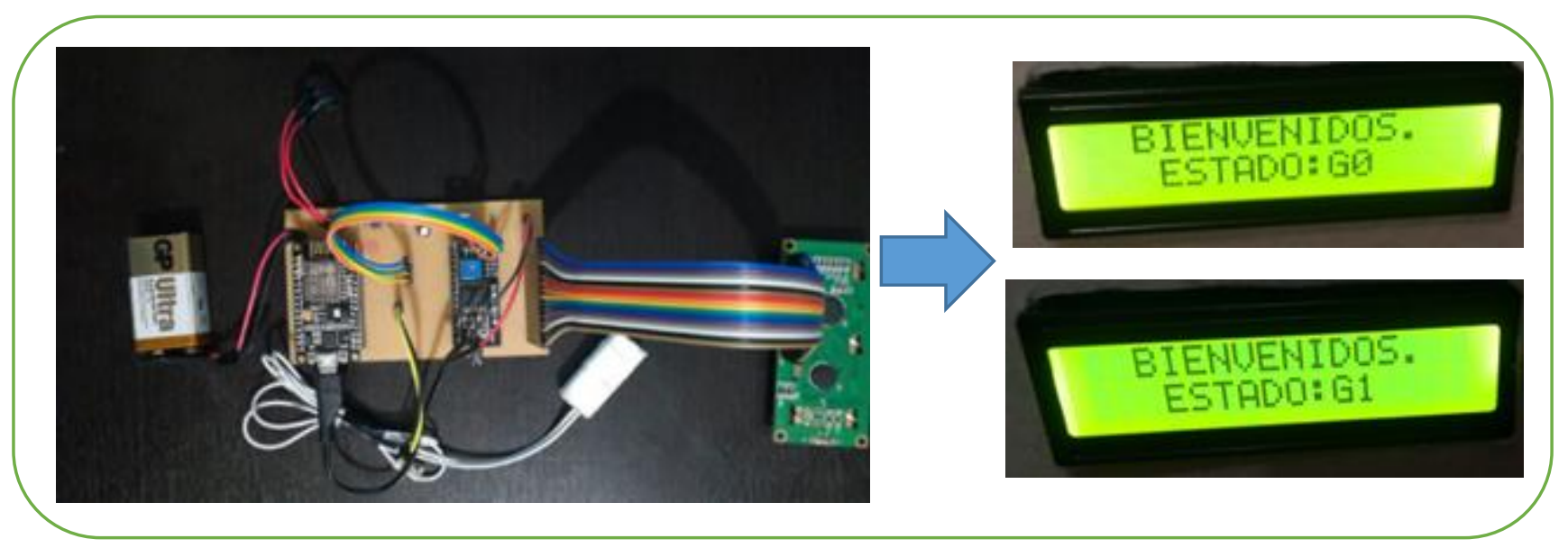

Fig. 14: Montaje PCB sensor magnético (Izquierdo), Estados LCD(Derecho).

Para el sensor de magnético de Movimiento. 


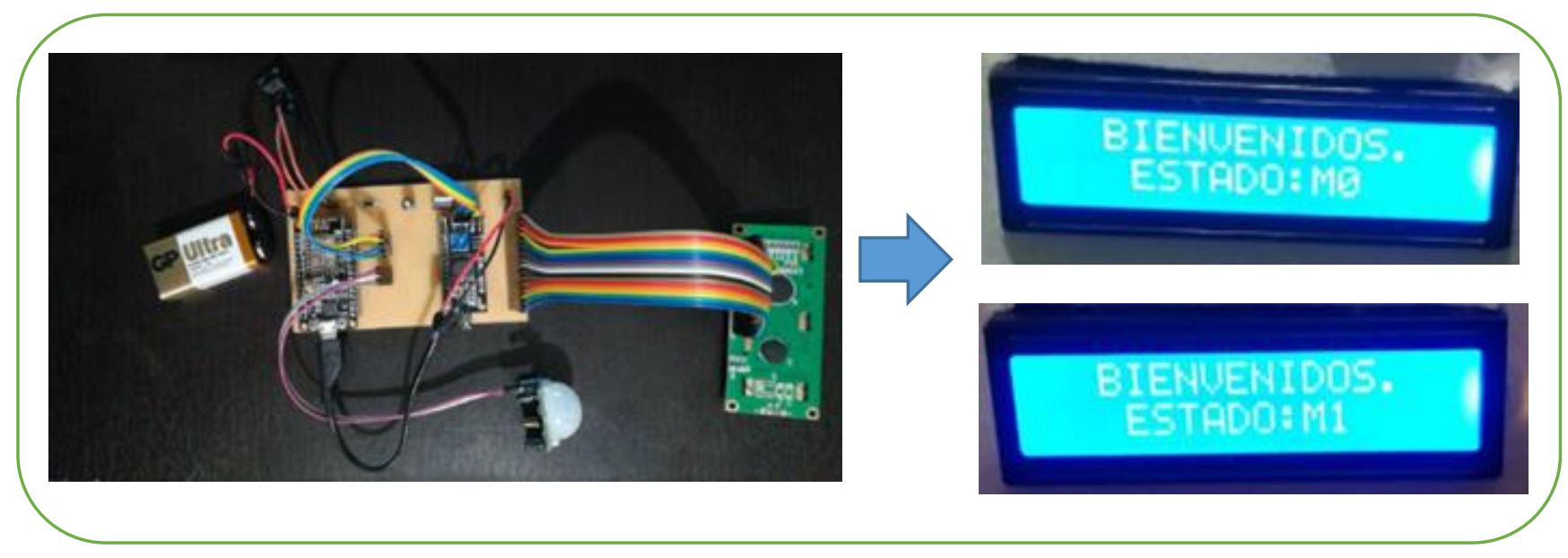

Fig. 15: PCB Sensor de movimiento.

Para el sensor de magnético de Corriente

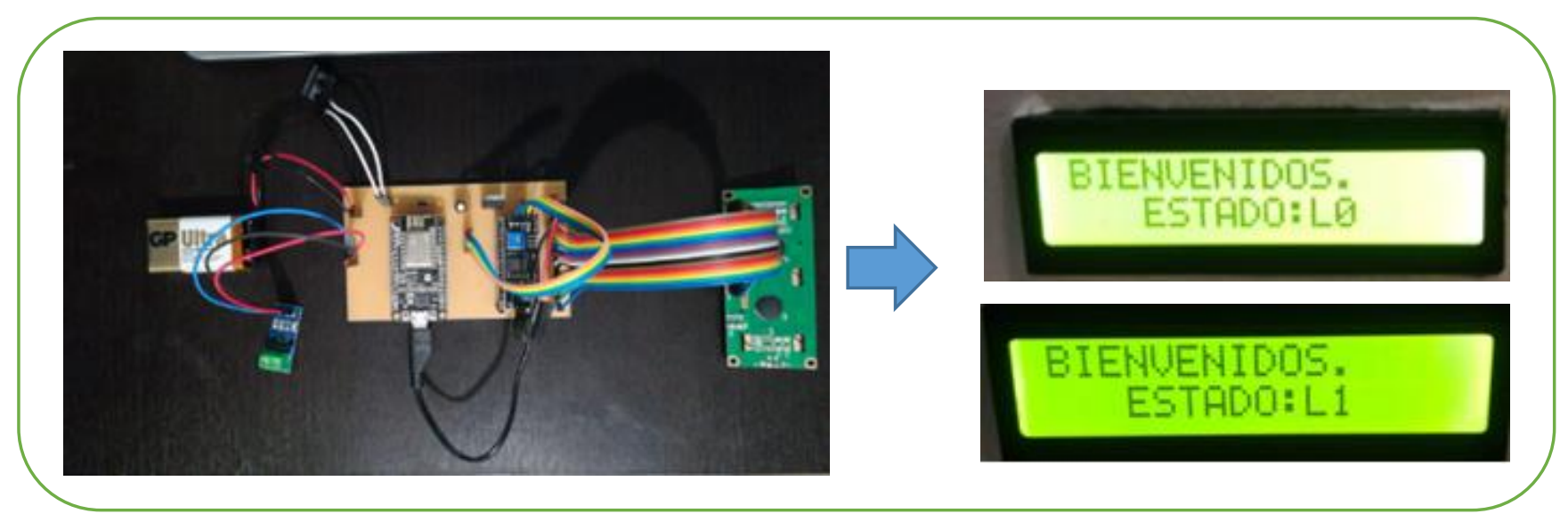

Fig. 16: PCB Sensor de corriente.

A cada sensor se le construyó una caja y posteriormente se instalaron todos los sensores en la cocina, como se observa en la figura 17.

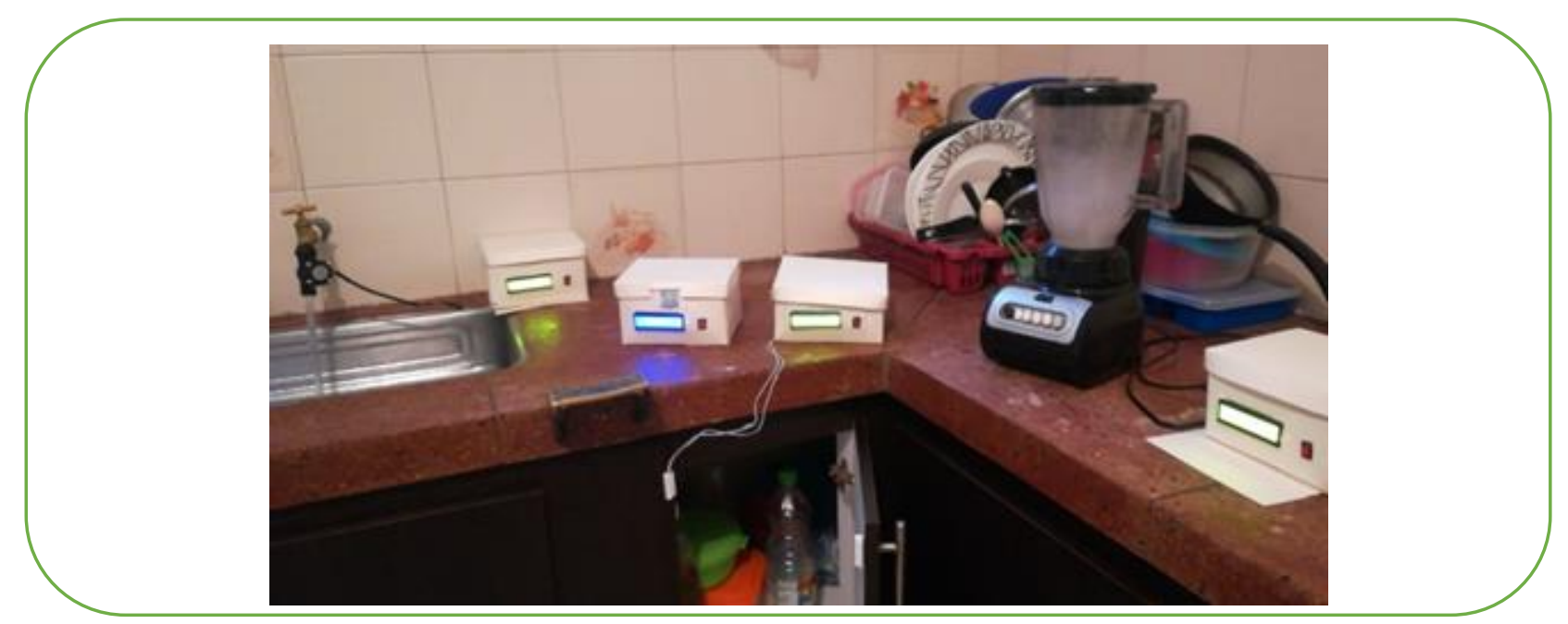

Fig. 17: Sensores en la cocina. 


\section{Paso 7. Conexión al servidor gratuito ThingSpeak}

A cada uno de los programas realizados para la programación del node, con el funcionamiento de la pantalla LCD, se le agregaron 2 librerías \#include <ESP8266WiFi.h>, la cual es con la que se establece la comunicación wifi y permite agregar el nombre de la red wifi, su respectiva contraseña, y la \#include $<$ ThingSpeak.h> para realizar la comunicación con el servidor, en ésta se agregan tanto la dirección IP, el número de identificación del canal, las claves de lectura y escritura, la dirección del campo y un retardo de 10 segundos para que cada vez que transcurre este tiempo el servidor obtenga un dato, tal y como se observa en las figuras 18 y 19.

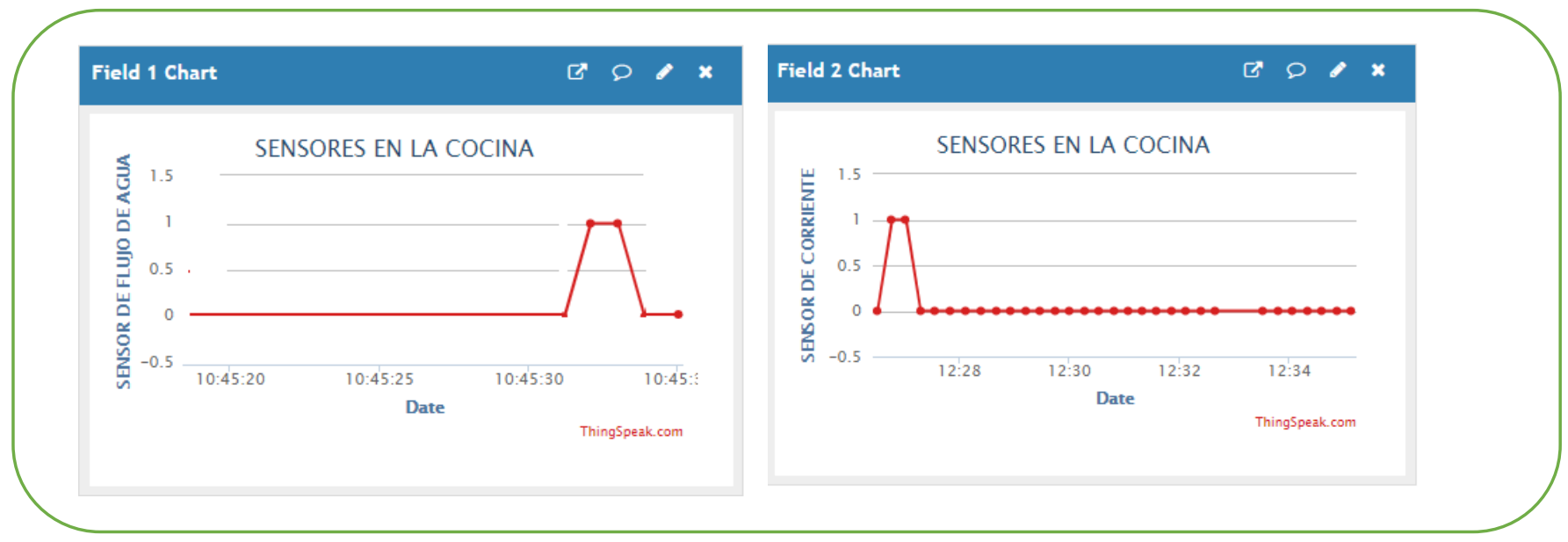

Fig. 18: Resultados estados sensor de flujo de agua (Izquierda) y sensor de corriente (Derecha).

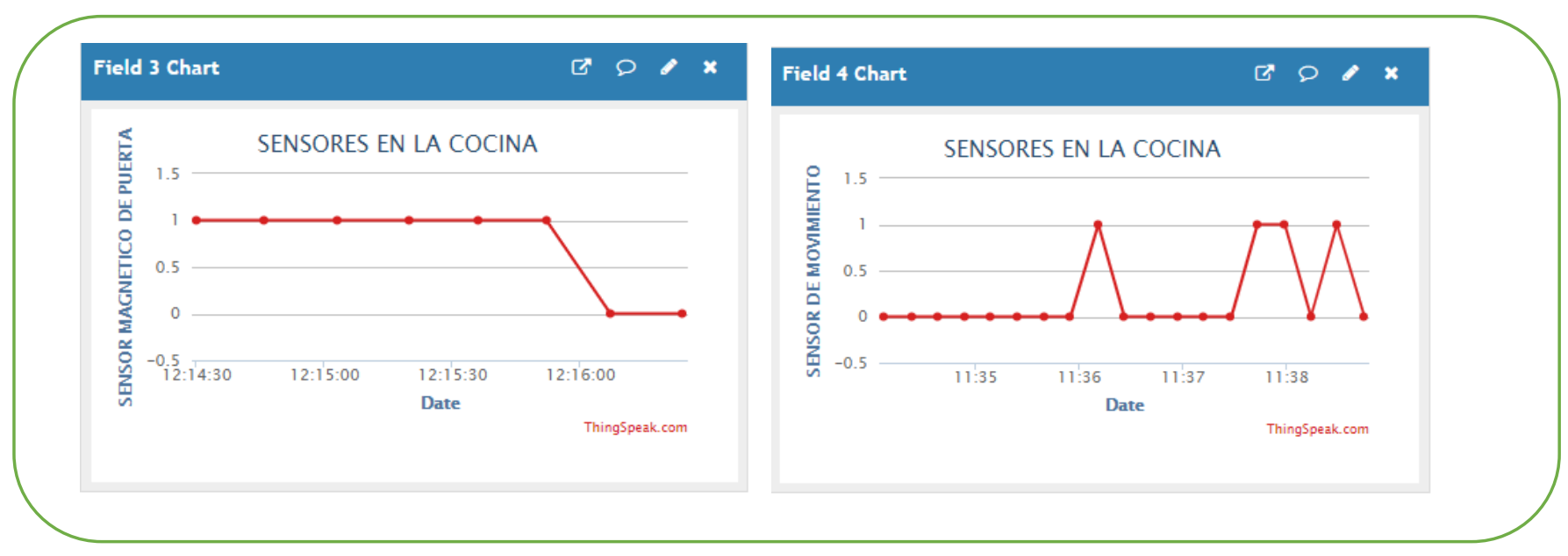

Fig. 19: Resultados estados sensor magnético de puerta (Izquierda) y sensor de movimiento (Derecha).

\section{DISCUSIÓN}

Respecto a la programación de los sensores, se observó que estos respondieron adecuadamente al objetivo del proyecto, el cual era usar los sensores para caracterizar la escena en la cocina; respecto al sensor magnético de puerta MC-38 se observó que es una herramienta eficaz y económica para identificar cuando una gaveta está abierta o cerrada; como se observó en la tabla 5 el porcentaje de efectividad es del 100\%; de igual manera este sensor puede ser usado en una puerta o ventana, con actuadores para abrirlas dependiendo de las necesidades del usuario; adicionalmente en el interior de cada gaveta se podrían colocar sensores de peso, o una cámara para el reconocimiento de objetos y tener certeza del interior de las gavetas.

En relación al sensor de corriente ACS-712 se pudo observar que es muy útil para determinar si un electrodoméstico está encendido o apagado, como se observó en la tabla 6 el porcentaje de efectividad es del 100\%; la desventaja de este sensor es que es invasivo, por lo cual se debe abrir el circuito o el cable de alimentación del electrodoméstico para poder medirla, como se observó en el montaje de la figura 10, al cual se le adaptó un extensión eléctrica (blanca) para abrir el circuito y así no dañar el cable de la licuadora; se debe buscar entonces otro tipo de sensor que sea no invasivo, o diseñar un propio módulo no invasivo; de 
igual manera como se observó en el diagrama de flujo de la tabla 2 sección B) este sensor permite obtener las mediciones de corriente IP, corriente IRMS y potencia, se podría usar en un registro de consumo.

En cuanto al sensor de flujo de agua YF-S201, se concluyó que es una herramienta muy eficaz para determinar si la llave del lavaplatos está abierta o cerrada, como se observó en la tabla 7, el cual demostró una efectividad del 100\%; de igual manera como desarrollo futuro, se le podría incorporar un actuador de forma que permita cerrar o abrir la llave de manera remota, así mismo como se evidenció en la figura 11; este sensor permite adicionalmente saber el caudal, el cual se podría usar para monitorear el consumo de agua.

En consideración al sensor de movimiento piroelectrico HC- SR501 éste traía consigo dos trimmers, uno para ajustar el tiempo entre mediciones y otro para el ajuste de sensibilidad de medición; se realizaron varias pruebas para ajustarlos adecuadamente, dado que en ocasiones, aunque había movimiento, no lo detectaba, pero gracias a esta calibración, se obtuvo un porcentaje de efectividad del $86,2 \%$ en el análisis de varianza, como se pudo evidenciar en la tabla 10, dado que el modelo no es significativo; sin embargo, este sensor de movimiento no tiene la capacidad de determinar quién realiza el movimiento, puede ser un niño que esté jugando en la cocina o una mascota, por lo cual sería de gran ayuda implementar un sistema más complejo, como el de reconocimiento de rostros asistido por nubes y Raspberry $\mathrm{Pi}$, dado que ya hay investigaciones enfocadas a utilizar esta tecnología con diferentes usos como ayudar a la policía a identificar criminales (Sajjad et al., 2017).

Respecto a las figuras $13,14,15$ y 16 donde se observa el montaje de cada sensor en las PCB, se concluye que las PCB quedaron bien diseñadas, dado que éstas funcionaron sin ningún inconveniente, pero como se puede observar en la figura 17, cuando se realizó la caja para proteger las PCB, se observó que éstas quedaron grandes, y para una implementación futura en una cocina real no sería conveniente, dado que ocupan mucho espacio, entonces como desarrollo futuro se podría realizar la miniaturización del circuito. En relación a la figura 18 parte izquierda, donde se encuentran los datos obtenidos por el sensor de flujo agua, se observa que tiene 59 puntos, por lo tanto equivale a 590 segundos, aproximadamente 10 minutos de muestreo, cuando la gráfica está en 0 significa que la llave del lavaplatos está cerrada y cuando está en 1 significa que está abierta, de la gráfica se observa que la llave estuvo abierta por 50 segundos, en el eje del tiempo (Date) nos permite ver la hora que fue activada aproximadamente 10:44 AM, adicionalmente aunque no se observa en las gráficas, al posicionarnos sobre cada punto, se puede observar la fecha; aunque el intervalo de tiempo fue pequeño, se puede observar el correcto funcionamiento del dispositivo creado. Como desarrollos futuros, convendría conectarlo durante un tiempo mayor, ya sea un día, una semana, o un mes, también se podrían obtener los datos como el caudal consumido y así realizar un análisis más detallado.

En consideración a la gráfica del sensor de corriente (Figura 17, parte derecha), se observa que tiene 11 puntos correspondientes a 110 segundos, lo que equivale a 1,86 min, si bien es un tiempo corto para analizar las tendencias de funcionamiento del sensor, sí se utiliza la licuadora en las mañanas, en la noche, o al medio día, como se observa en la gráfica, dado que en la escala de tiempo, muestra 12:27 AM, se evidencia el correcto funcionamiento del enlace hardware y software realizado en este artículo. De igual manera que en el caso anterior, realizar un análisis más profundo, a futuro se debería dejar conectado el sensor más tiempo, realizar un análisis con más datos, inclusive se podrían obtener modelos matemáticos acerca del funcionamiento de éstos. En cuanto a la figura 19 parte izquierda, relacionada con los datos obtenidos del sensor magnético de puerta, se observa que se tomaron 21 datos, cada uno representado por un punto sobre la gráfica, el cual equivale a 210 segundos, lo que corresponde aproximadamente a 3 minutos y medio, allí se evidencian 2 estados, 1 y 0 , el 1 equivale a la gaveta abierta, y el 0 a la gaveta está cerrada, así mismo se observa que el cambio de estado fue realizado en las horas de la tarde, aproximadamente 4:48 PM. Como desarrollo futuro, al igual que en los casos anteriores, se debe realizar la adquisición de datos por una cantidad de tiempo mayor, para así poder hacer un análisis más estructurado, y obtener modelos matemáticos que permitan predecir la escena en la cocina de manera más clara y precisa.

Respecto a la última gráfica correspondiente al sensor de movimiento, que se encuentra ubicada en la figura 19 parte derecha, observamos que se obtuvieron 19 datos, correspondientes a 190 segundos y 3.22 minutos aproximadamente, se nota que este sensor es muy sensible, dado que obtuvo cambios de estados muy continuos, al igual que en los casos anteriores, la gráfica muestra 2 estados, 0 cuando no hay movimiento y 1 cuando hay movimiento en la cocina. Como desarrollo futuro se podría buscar implementar otros tipos de sensores de movimiento que sean más estables, y de igual manera realizar una captura de datos por tiempos mayores, para realizar un análisis más detallado. Es de notar que la literatura para comparar el trabajo realizado es limitada, aunque existen investigaciones basadas en IoT, en áreas como la agricultura de precisión, para obtención, monitoreo y análisis de variables climáticas (Quiroga, et al., 2017), así como en la salud, para poder acceder a diversos servicios médicos a través de loT independientemente del estrato social (Mendoza et al., 2016) o en el impacto en el diagnóstico y rehabilitación de pacientes con deterioro cognitivo (Murillo et al., 2015). 


\section{CONCLUSIONES}

De los resultados mostrados, de su análisis y su discusión se puede concluir que: 1) El sensor de puerta Mc38 es una herramienta eficaz y económica para identificar cuando una gaveta o puerta está abierta o cerrada y tiene una efectividad del $100 \%$. 2) El sensor de corriente ACS712 es muy útil para determinar si un electrodoméstico está encendido o apagado, tiene una efectividad del $100 \%$ y permite realizar mediciones de corriente Ip e IRMS. 3) El sensor de flujo de agua YF-S201 permite conocer cuando una llave está abierta o cerrada, tiene una efectividad del $100 \%$ y determina su caudal. 4) El sensor de movimiento piroeléctrico HCSR501 tiene una efectividad del $86.2 \%$ con resultados muy estables según el análisis de varianza. 5) El diseño realizado en PCB para cada uno de los sensores funcionó al $100 \%$ dado que no hubo problemas con las conexiones y mostró adecuadamente en la pantalla LCD el estado de cada uno. 6) El análisis de la escena por medio de graficas obtenidas en el servidor ThingSpeak fue óptimo, dado que permitió ver la cantidad de datos adquiridos, un tiempo aproximado de duración de la adquisición de datos, una fecha exacta en la cual se realizaron los cambios.

\section{AGRADECIMIENTOS}

Se agradece la colaboración prestada, a la Universidad Nacional de Colombia -sede Manizales, Universidad de Caldas, MABE y a todos los integrantes del proyecto denominado: Prototipo computacional para la fusión y análisis de grandes volúmenes de datos en entornos iot (internet de las cosas) a partir de técnicas de machine learning y arquitecturas seguras entre sensores, para caracterizar el comportamiento e interacción de los usuarios en un ecosistema de connected home con código 36715.

\section{REFERENCIAS}

Amankwah, J. y S. Adomako, Big data analytics and business failures in data-Rich environments: An organizing framework, doi:10.1016/j.compind.2018.12.015, Computers in Industry, 105, 204-212 (2019)

Ardobot, Sensor Magnético para Puertas y Ventanas MC-38, Ardobot en Colombia (2018)

Arévalo, D., S. Nájera y E. Piñero, La Influencia de la Implementación de las Tecnologías de Información en la Productividad de Empresas de Servicios, doi: 10.4067/S071807642018000600199, Inf. Tecnol. 29(6), (2018)

Bayani, M., A. Segura, M. Alvarado y M. Loaiza, IoT-Based Library Automation and Monitoring system: Developing an Implementation framework of Implementation, doi: 10.15517/eci.v8i1.30010, E-Ciencias de la infomacion, 8(1), (2018)

Bogdan, R., B. Gerkey y M. Beetz, Robots in the kitchen: Exploiting Ubiquitous Sensing and Actuation, doi: 10.1016/j.robot.2008.06.010, Robotic and Autonomous Systems, 56, 844-856 (2008)

BrikoGeek, NodeMCu v3-ESP8266, BrikoGeek en España (2019)

Castro, D., W. Coral y otros cuatro autores, Survey on loT solutions applied to Healthcare, doi:10.15446/dyna.v84n203.64558, Dyna, 84(203), (2017)

Electronicapty, Sensor Magnético Mc-38 para puertas y ventanas, Electronicapty en Panamá (2017)

Gomez, C., F.Valencia y C. Marulanda, Las Tecnologías de la Información y las Comunicaciones y los Servicios Tecnológicos en las Entidades Públicas del Triángulo del Café en Colombia, doi: 10.4067/S0718-07642018000400119, Inf. Tecnol. 29(4), (2018)

Gonzales, C., C. Cárdenas y M. Mendoza, M2M system for efficient water consumption in sanitary services, based on intelligent environment, doi: 10.15446/dyna.v85n204.68264, Dyna, 85(204), (2018)

Innova didactic, Keyestudio Sensor de movimiento PIR, Innova didactic en Macedonia (2019)

Klein, A., F. Beal y R. Da rosa, Internet of things-based products/services: process and challenges on developing the business models, doi: 10.4301/s1807-17752017000300009, JISTEM J.Inf.Syst. Technol. Manag, 14(3), (2017)

Li, G., D. Zhang y otros 4 autores, A Kind of New Multicast Routing Algorithm for Application of Internet of Things, J. appl. res. Technol, ISSN: 2448-6736, 11(4), (2013)

Llamas, L., Detector de Movimiento con Arduino y Sensor Pir, Luis llamas en España (2015)

Maksimović, M., The roles of Nanotechnology and Internet of Nano things in healthcare transformation, TecnoL, ISSN: 2256-5337, 20(40) (2017)

Mendoza, P., K. Hernández, C. Vilora y D. Jabba, Internet de las cosas y la salud centrada en el hogar, Revista Salud Uninorte, ISSN: 2011-7531, 32(2), (2016)

Moviltronics, Sensor de Movimiento PIR HC-SR501, Moviltronics en Colombia (2019)

Moviltronics, Sensor de Movimiento PIR mini HC-SR505, Moviltronics en Colombia (2019)

Murillo, S., C. Marquez y B. Segura, Impacto de loT en la prevención, asistencia, detección y rehabilitación de pacientes con deterioro cognitivo: una revisión, Entre Ciencia e Ingenieria, ISSN: 1909-8367, 9(17), (2015) 
Naylampmechatronics, Tutorial Sensor de Corriente ACS712, Naylampmechatronics en Perú (2016)

Naylampmechatronics, Tutorial Sensor de Flujo de Agua, Naylampmechatronics en Perú (2016)

Oliveira, E., R. Alfaia y otros 4 autores, SmartCoM: Smart Consumption Management Architecture for Providing a UserFriendly Smart Home based on Metering and Computational Intelligence, doi:10.1590/2179-10742017v16i3965, J. Microw. Optoelectron. Electromagn. Appl, 16(3), (2017)

Parallax, PIR sensor with LED signal, Parallax en USA (2019)

Parsch, P. y A. Masrur, On Reliable Communication in Transmit-only Networks for Home Automation, doi: 10.1016/j.jnca.2017.10.023, Journal of Network and Computer Applications, 101, 96-110 (2017)

Pia, S., A. Van Deursen y T. Van Rompay, Accepting the Internet-of-Things in our homes: The role of user skills, doi: 10.1016/j.tele.2018.12.004, Telematics and Informatics, 36, 147-156 (2019)

Plasencia, L. y C. Anias, Arquitectura referencial de Big Data para la gestión de las telecomunicaciones, doi:10.4067/S0718-33052017000400566, Ingeniare. Rev. chil. Ing. 25(4), (2016)

Quiroga, E., S. Jaramillo, W. Campo y G. Chanchi, Propuesta de una Arquitectura para Agricultura de Precisión Soportada en loT, doi:10.17013/risti.24.39-56, RISTI, 24, (2017)

Ray. P.P., A survey on Internet of Things architectures, doi: 10.1016/j.jksuci.2016.10.003, Journal of King Saud University - Computer and Information Sciences, 30(3), 291-319 (2018)

Rodríguez F.J., Automatización Mediante Equipos EIB de una Cocina Adaptada Dentro del Entorno del Robot Asistencial ASIBOT, Proyecto Fin de Carrera, Departamento de Ingeniería de sistemas y automática, U. Carlos III, Madrid (2010)

Sajjad, M., M. Nasir y otros 6 autores, Raspberry Pi Assisted Face Recognition Framework for Enhanced Law-Enforcement Services in Smart Cities, doi: 10.1016/j.future.2017.11.013, In Press, Corrected Proof (2017)

Sanchez, M. y G. Ramoscelli, Value creation from the Internet of things: Exploratory study in Buenos Aires Province, Vis. Futuro, ISSN: 1669-7634, 22(1), (2018)

Sitrack, Blog: Esto es lo que se espera del loT para el 2020, Pablo Perinetti (2017)

Tdrobotica, Arduino básico T18AB, Tdrobotica (2011)

Teixeira, F., F. Pereira, H.C. Wong, J. Nogueira y L. Oliveira, SloT: Securing Internet of Things through distributed systems analysis, doi: 10.1016/j.future.2017.08.010, Future Generation Computer Systems, 92, 1172-1186 (2019)

Toloza, C., Automatización de Cocina (2016)

Vidal, C., M. Bustamante, M. Lapo y M. Nuñez, En la Búsqueda de Soluciones MapReduce Modulares para el Trabajo con BigData: Hadoop Orientado a Aspectos, doi: 10.4067/S0718-07642018000200133, Inf. Tecnol. 29(2), (2018)

Wang, Z.L., Entropy theory of distributed energy for Internet of Things, doi: 10.1016/j.nanoen.2019.02.012, Nano Energy, $58,669-672(2019)$ 